\title{
Guidelines for Mechanical Thrombectomy in Japan, the Fourth Edition, March 2020: A Guideline from the Japan Stroke Society, the Japan Neurosurgical Society, and the Japanese Society for Neuroendovascular Therapy
}

\author{
Hiroshi Yamagami, ${ }^{1}$ Mikito HayaKawa, ${ }^{2}$ Manabu InOUE, ${ }^{3}$ Koji IiHaRA, ${ }^{4}$ \\ Kuniaki OGASAWARA, ${ }^{5}$ Kazunori TOYODA, ${ }^{6}$ Yasuhiro HASEGAWA,, 8 \\ Kenji OHATA, ${ }^{9}$ Yoshiaki SHIOKAWA, ${ }^{10}$ Kazuhiko NOZAKI, ${ }^{11}$ \\ Masayuki EZURA, ${ }^{12}$ Toru IWAMA, ${ }^{13}$ \\ and JSS/JNS/JSNET Joint Guideline Authoring Committee
}

${ }^{1}$ Department of Stroke Neurology, National Hospital Organization Osaka National Hospital, Osaka, Osaka, Japan

${ }^{2}$ Division of Stroke Prevention and Treatment, Faculty of Medicine, University of Tsukuba, Tsukuba, Ibaraki, Japan

${ }^{3}$ Division of Stroke Care Unit/Department of Cerebrovascular Medicine, National Cerebral and Cardiovascular Center, Suita, Osaka, Japan

${ }^{4}$ Department of Neurosurgery, National Cerebral and Cardiovascular Center,

Suita, Osaka, Japan

${ }^{5}$ Department of Neurosurgery, Iwate Medical University, Morioka, Iwate, Japan

${ }^{6}$ Department of Cerebrovascular Medicine, National Cerebral and Cardiovascular Center, Suita, Osaka, Japan

${ }^{7}$ Department of Neurology, St. Marianna University School of Medicine, Kawasaki, Kanagawa, Japan

${ }^{8}$ Stroke Center and Department of Neurology, Shin-yurigaoka General Hospital, Kawasaki, Kanagawa, Japan

${ }^{9}$ Department of Neurosurgery, Osaka City University Graduate School of Medicine, Osaka, Osaka, Japan

${ }^{10}$ Department of Neurosurgery, Kyorin University, Mitaka, Tokyo, Japan

${ }^{11}$ Department of Neurosurgery, Shiga University of Medical Science, Otsu, Shiga, Japan

${ }^{12}$ Department of Neurosurgery, National Hospital Organization Sendai Medical Center,

Sendai, Miyagi, Japan

${ }^{13}$ Department of Neurosurgery, Gifu University Graduate School of Medicine, Gifu, Gifu, Japan 


\section{Table of Contents}

List of Abbreviations 165

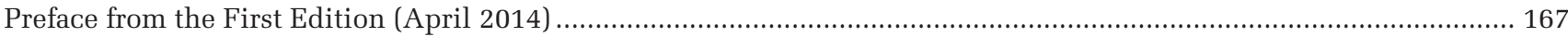

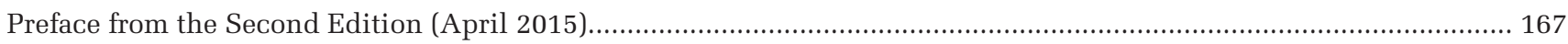

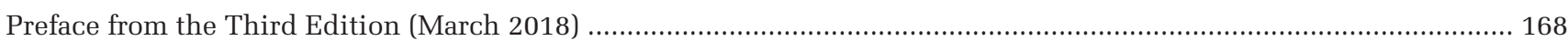

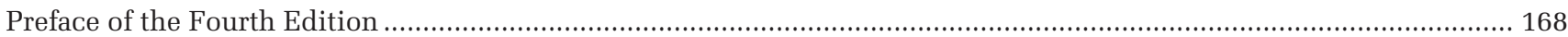

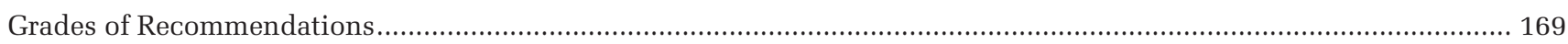

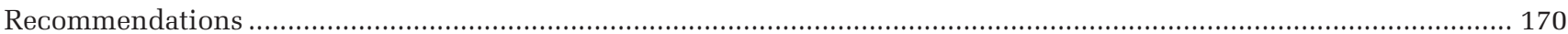

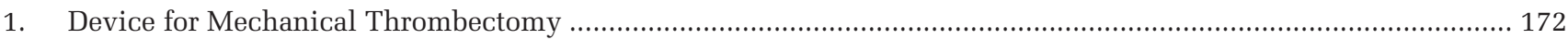

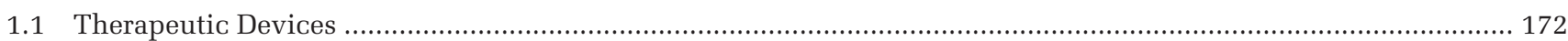

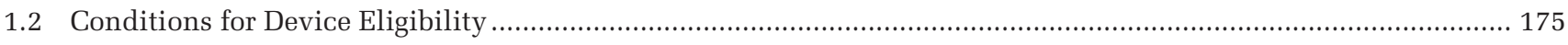

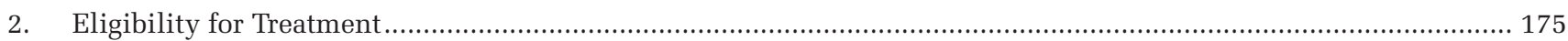

2.1 Randomized Controlled Trials of Mechanical Thrombectomy …............................................................. 175

2.2 Eligibility Based on Diagnostic Imaging and Neurological Deficits ......................................................... 179

2.3 Patients in Whom the Efficacy Is Not Established .................................................................................... 180

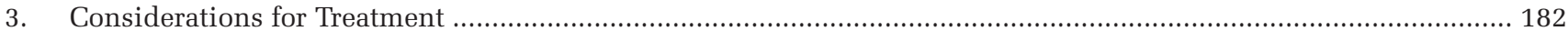

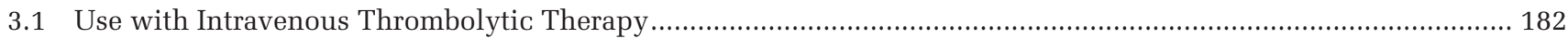

3.2 Times from Onset to Arterial Puncture and Reperfusion ....................................................................... 183

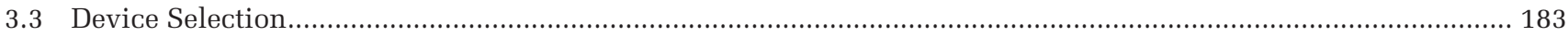

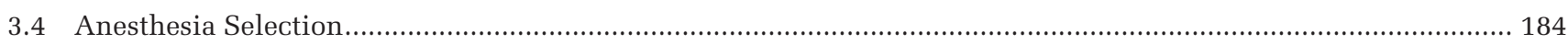

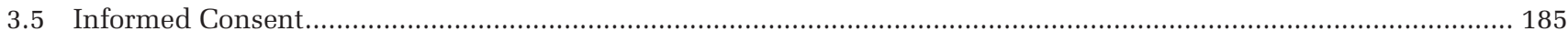

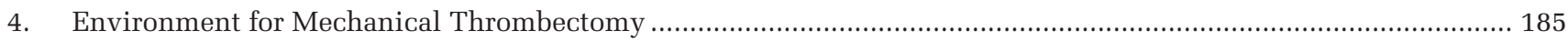

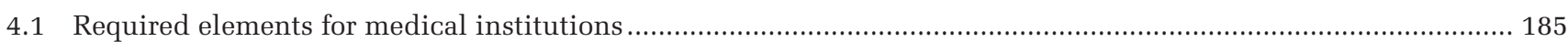

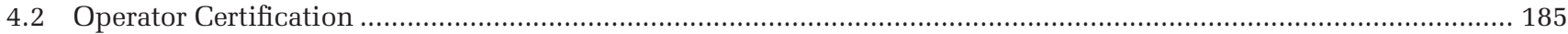

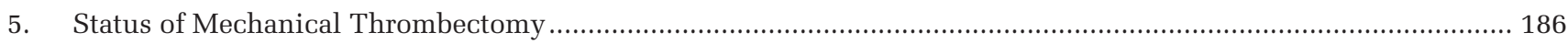

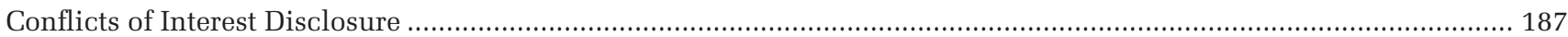

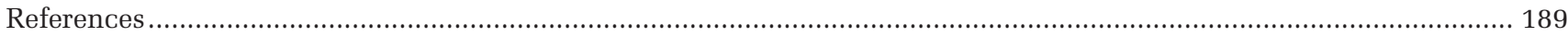




\section{List of Abbreviations}

\section{Common noun}

\begin{tabular}{|c|c|}
\hline Abbreviation & Formal name \\
\hline ACA & anterior cerebral artery \\
\hline ADAPT & a direct aspiration first-pass technique \\
\hline ADC & apparent diffusion coefficient \\
\hline BA & basilar artery \\
\hline CIM & clinical imaging mismatch \\
\hline CT & computed tomography \\
\hline СTP & computed tomography perfusion \\
\hline DWI & diffusion-weighted imaging \\
\hline FLAIR & fluid-attenuated inversion recovery \\
\hline ICA & internal carotid artery \\
\hline ITT & intention to treat \\
\hline IV & intravenous \\
\hline MCA & middle cerebral artery \\
\hline MT & mechanical thrombectomy \\
\hline MRA & magnetic resonance angiography \\
\hline MRI & magnetic resonance imaging \\
\hline $\mathrm{mRS}$ & modified Rankin scale \\
\hline NCCT & non-contrast computed tomography \\
\hline NIHSS & National Institutes of Health Stroke Scale \\
\hline PCA & posterior cerebral artery \\
\hline RCT & randomized controlled trial \\
\hline rt-PA & recombinant tissue-plasminogen activatorSICH \\
\hline $\mathrm{SICH}$ & symptomatic intracranial hemorrhage \\
\hline TICI & Thrombolysis in Cerebral Infarction \\
\hline TIMI & Thrombolysis in Myocardial Infarction \\
\hline VA & vertebral artery \\
\hline
\end{tabular}

Trials, organization, etc.

\begin{tabular}{|l|l|}
\hline Abbreviation & \multicolumn{1}{|c|}{ Formal name } \\
\hline AHA & the American Heart Association \\
AnStroke & Anesthesia During Stroke \\
ASA & the American Stroke Association \\
ASPECTS & Alberta Stroke Programme Early CT Score \\
ASTER & Contact Aspiration vs. Stent Retriever for Successful Revascularization \\
BASILAR & EVT for Acute Basilar Artery Occlusion Study \\
BEST & Basilar Artery Occlusion Endovascular Intervention versus Standard Medical Treatment \\
COMPASS & Comparison of Direct Aspiration vs. Stent Retriever as a First Approach \\
DAWN & DWI or CTP Assessment with Clinical Mismatch in the Triage of Wake-Up and Late \\
& Presenting Strokes Undergoing Neurointervention with Trevo \\
ECASS & European Cooperative Acute Stroke Study \\
\hline
\end{tabular}




\begin{tabular}{|c|c|}
\hline ESCAPE & $\begin{array}{l}\text { Endovascular Treatment for Small Core and Anterior Circulation Proximal Occlusion } \\
\text { with Emphasis on Minimizing CT to Recanalization Times }\end{array}$ \\
\hline EXTEND-IA & EXtending the time for Thrombolysis in Emergency Neurological Deficits-Intra-Arterial \\
\hline $\begin{array}{l}\text { EXTEND-IA } \\
\text { TNK }\end{array}$ & Thrombolysis in Emergency Neurological Deficits-Intra-Arterial using Tenecteplase \\
\hline GOLIATH & General or Local Anesthesia in Intra Arterial Therapy \\
\hline HERMES & Highly Effective Reperfusion evaluated in Multiple Endovascular Stroke Trials \\
\hline IMS & Interventional Management of Stroke \\
\hline J-ACT & Japan Alteplase Clinical Trial \\
\hline JNS & the Japan Neurosurgical Society \\
\hline JSNET & the Japanese Society for Neuroendovascular Therapy \\
\hline JSS & the Japan Stroke Society \\
\hline MR CLEAN & $\begin{array}{l}\text { Multicenter Randomized Clinical Trial of Endovascular Treatment for Acute Ischemic } \\
\text { Stroke in The Netherlands }\end{array}$ \\
\hline MR-RESCUE & Mechanical Retrieval and Recanalization of Stroke Clots Using Embolectomy \\
\hline NINDS & National Institute of Neurological Disorders and Stroke \\
\hline PISTE & Pragmatic Ischaemic Stroke Thrombectomy Evaluation \\
\hline REVASCAT & $\begin{array}{l}\text { Randomized Trial of Revascularization with Solitaire FR Device versus Best Medical } \\
\text { Therapy in the Treatment of Acute Stroke Due to Anterior Circulation Large Vessel } \\
\text { Occlusion Presenting within Eight Hours of Symptom Onset }\end{array}$ \\
\hline RIVER & Reperfuse Ischemic Vessels with Endovascular Recanalization device \\
\hline SIESTA & Sedation vs. Intubation for Endovascular Stroke Treatment \\
\hline SWIFT & Solitaire FR With the Intention For Thrombectomy \\
\hline SWIFT PRIME & $\begin{array}{l}\text { Solitaire FR With the Intention For Thrombectomy as PRIMary Endovascular } \\
\text { Treatment for Acute Ischemic Stroke }\end{array}$ \\
\hline THRACE & THRombectomie des Artères CErebrales \\
\hline WAKE-UP & Efficacy and Safety of MRI-Based Thrombolysis in Wake-Up Stroke \\
\hline
\end{tabular}




\section{Preface from the Guidelines for Mechanical Thrombectomy in Japan, the First Edition (April 2014)}

Acute cerebral artery occlusion is associated with very poor outcomes. ${ }^{1)}$ Early recanalization of occluded arteries, however, may improve the outcome, and recanalization therapy has been attempted with a variety of approaches. The most well-known approach is intravenous therapy with the recombinant tissue-plasminogen activator (IV rt-PA), alteplase. The efficacy of IV rt-PA has been established through many randomized controlled trials (RCTs).,23) IV rt-PA is classified as a Class I therapy in the Guidelines for the Early Management of Patients with Acute Ischemic Stroke of the American Heart Association/American Stroke Association (AHA/ASA) ${ }^{4}$ and a Grade A recommendation in the Japanese Guidelines for the Management of Stroke. ${ }^{5)}$ Even as cerebrovascular medical technology has progressed, IV rt-PA remains the priority therapy for patients with acute ischemic stroke. Currently, IV rt-PA is indicated for acute ischemic stroke within 4.5 hours of onset with no contraindications. ${ }^{6,7)}$

Endovascular treatment, made possible with the development of microcatheters capable of intracranial access, represents another approach that enables effective local intra-arterial fibrinolysis for middle cerebral artery (MCA) occlusion within 6 hours of onset. ${ }^{8,9)}$ The Merci Retriever (the then Concentric Medical Inc. ${ }^{10,11)}$ and the Penumbra System (Penumbra, Inc. ${ }^{12,13)}$ were subsequently developed for mechanical thrombectomy (MT). In patients contraindicated for or not responding to IV rt-PA, MT provides a significantly better outcome when recanalization is achieved compared with no recanalization and is therefore starting to be widely used in clinical settings. The recently developed stent retrievers Solitaire FR (Covidien) and Trevo (Stryker Corporation) have safety and efficacy profiles superior to the Merci Retriever. ${ }^{14,15)}$

The year 2013 has seen a stream of studies comparing IV rt-PA with endovascular therapy, evaluating the efficacy of diagnostic imaging for selecting recanalization therapy, and evaluating the efficacy of adding endovascular therapy to IV rt-PA. ${ }^{16-19)}$ These studies, however, could not show MT to be more effective than standard of care or IV rt-PA.

In Japan, the Merci Retriever in April 2010 and the Penumbra System in June 2011 were approved for use in MT. All-patient postmarketing surveillance was conducted over the 3 years following approval, with medical societies and others reporting some of the surveillance results. The Solitaire FR in December 2013 and the Trevo ProVue in March 2014 were approved as stent retrievers. A clinical trial of the REVIVE was conducted from 2013 to 2014, and approval is now pending.

The Japan Stroke Society (JSS), Japan Neurosurgical Society (JNS), and Japanese Society for Neuroendovascular Therapy (JSNET) are actively involved in MT, and they created the "Guidelines for Mechanical Thrombectomy in Japan" to coincide with the approval of the Merci Retriever and the Penumbra System. These Guidelines, which lay out a training program for operators satisfying coursework requirements, advocate for appropriate device use in MT. We decided to create and publish new guidelines for appropriate use in MT to address the growing body of knowledge about this procedure and because new devices are expected to become available. We hope that MT operators will provide MT as indicated and using the proper technique based on a full understanding of the information contained in these Guidelines.

\section{Preface from the Guidelines for Mechanical Thrombectomy in Japan, the Second Edition (April 2015)}

These Guidelines, established by the JSS, JNS, and JSNET, were first published in April 2014 (see above). Soon thereafter, the results of 4 RCTs were released in succession (MR CLEAN ${ }^{20)}$ in October 2014 and ESCAPE, ${ }^{21)}$ EXTEND-IA, ${ }^{22)}$ and SWIFT PRIME ${ }^{23)}$ in February 2015). These four studies showed that adding endovascular therapy primarily with stent retrievers to medical therapy including IV rt-PA for acute ischemic stroke caused by large artery occlusion of the anterior circulation (internal carotid artery [ICA] or proximal MCA) significantly improved independence in daily activities at 90 days compared with medical therapy alone. These studies, which demonstrated a certain level of efficacy and safety of endovascular therapy with MT devices in acute ischemic stroke, have had a major impact.

In response to these studies, JSS, JNS, and JSNET decided to revise the "Guidelines for Mechanical Thrombectomy in Japan" and publish the second edition as soon as possible. Our aim was to assist MT operators to properly select eligible patients and provide MT using proper techniques based on a full understanding of the information contained in these Guidelines. 


\section{Preface from the Guidelines for Mechanical Thrombectomy in Japan, the Third Edition (March 2018)}

Soon after the JSS, JSN, and JSNET released these Guidelines in April 2014, the results of four RCTs (MR CLEAN, ${ }^{20)}$ ESCAPE, ${ }^{21)}$ EXTEND-IA,22) and SWIFT PRIME23) were published. These studies showed that adding endovascular therapy primarily with stent retrievers to medical therapy including IV rt-PA for acute ischemic stroke caused by large artery occlusion of the anterior circulation (ICA or proximal MCA) significantly improved independence in daily activities at 90 days compared with medical therapy alone. In response to these study results, the JSS, JSN, and JSNET decided to revise the "Guidelines for Mechanical Thrombectomy in Japan" as quickly as possible, ultimately publishing the second edition in April 2015.

The findings of other controlled trials of MT for early acute ischemic stroke continued to be published, and knowledge continued to accumulate after the second edition was released. In 2018, the results of two RCTs in patients with acute ischemic stroke beyond 6 hours since the time last known well were published. These RCTs compared endovascular therapy plus medical therapy to medical therapy alone in patients with acute ischemic stroke caused by occlusion of the ICA or M1 segment of the MCA who had a mismatch between neurological deficits or the hypoperfusion area and ischemic core volume, with the DAWN trial ${ }^{24}$ enrolling patients with a time last known well of 6-24 hours, including those with an unknown time of onset, and the DEFUSE3 trial ${ }^{25)}$ enrolling patients with 6-16 hours from time last known well. In both studies, endovascular therapy improved modified Rankin Scale (mRS) scores after 3 months in late time windows. Although the types of image analysis software used in these studies are not widely used in Japan, they demonstrated a certain level of efficacy and safety of endovascular therapy with MT devices in selected patients, even in the cases presenting late.

Given the substantial impact of these studies, the JSS, JSN, and JSNET decided to revise the "Guidelines for Mechanical Thrombectomy in Japan" and release the third edition as soon as possible. Given the enormous amount of knowledge accumulated about MT, operators shall properly select eligible patients and provide MT using the proper technique based on a full understanding of the information contained in these Guidelines.

\section{Preface of the Guidelines for Mechanical Thrombectomy in Japan, the Fourth Edition}

These Guidelines, created by the JSS, JSN, and JSNET, were first published in April 2014. In response to the publication of the results of new RCTs and the accumulation of further knowledge, the JSS, JSN, and JSNET decided to revise the "Guidelines for Mechanical Thrombectomy in Japan" as quickly as possible, publishing the second edition in April 2015 and the third edition in March 2018.

After the release of the third edition, new stent retrievers and aspiration catheters for MT were approved and became eligible for insurance reimbursement. Moreover, serious attempts to combine multiple medical devices began, and even more knowledge was accumulated.

In December 2018, Japan's legislature passed the "Stroke and Cardiovascular Disease Control Act," which went into effect in December 2019. This act specifies criteria requiring physicians with experience consistent with neuroendovascular specialists capable of performing MT, as well as practice guidelines, to enable equal access to IV rt-PA in medical institutions nationwide and ensure that MT is widely available to eligible patients.

In response to these developments, the JSS, JSN, and JSNET decided to revise the "Guidelines for Mechanical Thrombectomy in Japan" and publish the fourth edition. As the body of knowledge about MT continues to grow, we hope that MT operators will properly select eligible patients and provide MT using the proper technique based on a full understanding of the information contained in these Guidelines. 


\section{Grades of Recommendations}

Grade of recommendation

\begin{tabular}{ll}
\hline Grade A & Strongly recommended \\
Grade B & Recommended \\
Grade C1 & May be considered, lacks sufficient scientific evidence \\
Grade C2 & Not recommended because of a lack of scientific evidence \\
Grade D & Not recommended because of potential harm \\
\hline
\end{tabular}

The classification of grading of treatment recommendations adhered to those presented in the 2015 Guidelines for the Management of Stroke (Supplement 2019). ${ }^{26)}$ 


\section{Recommendations}

\section{Device for MT}

1.1 MT should be provided using an approved device [Grade A]

The MT devices approved and in use in Japan at the time of the creation of these Guidelines were the stent retrievers Solitaire, Trevo, Tron FX, and EmboTrap and the aspiration catheters Sofia, AXS Catalyst, Penumbra, and REACT, as well as the Versi Retriever in a clinical trial.

1.2 Medical devices should be used in accordance with their approval conditions

The above MT devices are approved for use in achieving recanalization in patients with acute ischemic stroke within 8 hours of onset for whom IV rt-PA is not indicated or no recanalization was achieved with IV rt-PA. The indications for the Trevo and Solitaire were expanded in 2019 to include use within 24 hours of onset or the time last known well in patients who met certain imaging criteria. These devices are used to reduce damage by removing cerebral vessel thrombi to restore blood flow in acute ischemic stroke patients with relatively small infarcts obstructing the proximal major cerebral arteries of the anterior circulation (ICA or the M1 segment of the MCA). These devices were approved under the condition that endovascular therapy be started within 6-24 hours of the time last known well in patients for whom IV rt-PA is not indicated or who do not respond to IV rt-PA.

This approval condition does not necessarily agree with scientific evidence from clinical research on MT. Newly approved medical devices should be used in accordance with their approval conditions and specifications.

\section{Eligibility for Treatment}

Scientific evidence supports the effectiveness of MT for acute ischemic stroke caused by large vessel occlusion. MT is recommended with the following eligibility conditions.

1) For early acute ischemic stroke, endovascular therapy (including MT) with a stent retriever or aspiration catheter within 6 hours of onset in addition to medical therapy that includes IV rt-PA (alteplase) is recommended for patients who meet all of the following: (1) have been diagnosed with occlusion of a major cerebral artery of the anterior circulation (ICA or MCA M1 segment); (2) have a premorbid mRS score of 0 or 1; (3) have an Alberta Stroke Programme Early CT Score (ASPECTS) of $\geq 6$ on head CT or MRI diffusion-weighted imaging (DWI); (4) have a National Institutes of Health Stroke Scale (NIHSS) score of $\geq 6$; and (5) are at least 18 years of age [Grade A].

2) For ischemic stroke that appears to be caused by acute occlusion of the ICA or MCA M1 segment beyond 6 hours since the time last known well, MT within 16 hours of the time last known well is strongly recommended in patients with a premorbid mRS score of 0 or 1 , an NIHSS score of $\geq 10$, and ASPECTS of $\geq 7$ on MRI DWI [Grade A]. In addition, MT within 24 hours of the time last known well time is recommended for patients with a mismatch between ischemic core volume (CT perfusion or MRI DWI) and neurological deficits or a hypoperfusion lesion on perfusion imaging [Grade B].

However, in Japan, it should be noted that the intended use listed in the package insert of all thrombectomy devices other than the Trevo and Solitaire involves "acute ischemic stroke within 8 hours of onset."

3) Although substantial scientific evidence for MT is not available in patients with a large ischemic core having an ASPECTS of $<6$, mild neurological deficits with an NIHSS score of $<6$, acute occlusion of the MCA M2 segment or basilar artery (BA), or in patients with a premorbid mRS score of $\geq 2$, MT may be considered if careful consideration of the individual patient's condition shows that the efficacy benefits outweigh the safety risks [Grade C1].

4) Automated image analysis software capable of rapidly measuring ischemic core volume and detecting hypoperfusion lesions can be used for decision-making in MT when perfusion imaging would not be time-consuming [Grade C1].

\section{Considerations for Treatment}

3.1 IV rt-PA should be given priority in eligible patients [Grade A]

Based on accumulated scientific evidence, IV rt-PA is strongly recommended for eligible acute ischemic stroke patients within 4.5 hours of onset who have no contraindications. As in the Guidelines for Intravenous Thrombolysis (Recombinant Tissue-type Plasminogen Activator), the Third Edition, by the Japan Stroke Society, ${ }^{27,28)}$ IV rt-PA should be administered after carefully selecting eligible patients, and MT 
without IV rt-PA in eligible patients must be avoided in non-trial settings because the efficacy and safety of this approach had not been established as of the creation of these Guidelines.

Even when the time of onset is unknown, IV rt-PA may be considered within 4.5 hours of symptom recognition if DWI/fluid-attenuated inversion recovery (FLAIR) mismatch is present. However, the efficacy and safety profiles of IV rt-PA followed by thrombectomy have not been established in such patients who have large vessel occlusion [Grade C1].

3.2 Early treatment initiation and shorter times to recanalization are associated with better outcomes. Endovascular therapy (MT) should therefore be performed as soon as possible after the patient arrives [Grade A]. When IV rt-PA has been administered, the start of MT must not be delayed for the evaluation of thrombolysis effectiveness [Grade D].

3.3 As first-line devices for MT, stent retrievers and aspiration catheters have been shown to have comparable efficacy [Grade A]. Although a combined technique with a stent retriever and an aspiration catheter may be considered, it must be realized that this technique has not been shown to produce superior outcomes, involves complicated techniques, and incurs high costs [Grade C1].

3.4 MT is generally performed under local anesthesia. General anesthesia may be considered if medically required or if it can be provided without delaying the treatment [Grade C1].

3.5 The benefits and risks for MT should be explained and informed consent obtained from eligible patients or their legal representatives.

\section{Required elements for medical institutions}

4.1 The site should have an environment that allows IV rt-PA and should be equipped with an angiosuite that allows MT to be performed at any time [Grade A].

IV rt-PA is performed at sites that are capable of head CT or MRI, general hematology tests, coagulation tests, and electrocardiography; have an acute stroke care specialist able to begin treating patients as soon as possible after arrival; and have a system in place to rapidly provide neurosurgical intervention if needed.

Additionally, each site should have an environment that enables MT, that is, be equipped with an angiography room or an angio-suite in operating rooms, have an acute stroke care specialist able to begin the treatment as soon as possible after arrival, and continually monitor and improve their sites' environment.

4.2 MT must be performed by a specialist in neuroendovascular therapy or by a certified MT operator with comparable experience.

To ensure safe MT practice, this procedure must be performed by a specialist in neuroendovascular therapy certified by the JSNET or by a certified MT operator with comparable experience. The term "certified MT operator" refers to a member of the JSNET who is specialized in one of four core disciplines (i.e., neurosurgery, internal medicine, radiology, and emergency medicine), has performed diagnostic cerebral angiography on 200 patients, has performed neuroendovascular therapy on 100 patients (including 20 as the first operator and 15 as a provider of MT), and is registered by the JSNET.

\section{Status of MT}

Sites and operators should actively participate in surveillance/research on MT, helping to characterize the effects and problems of the procedure and assisting to build systems for providing care.

The practice of reperfusion therapy must be evaluated, and its safety and efficacy must be characterized through postmarketing surveillance and other clinical research on new devices. Assistance is needed for surveillance and research, including the grading of MT within therapies for acute cerebral artery occlusion.

Sites and practitioners must fulfill their roles and build systems for providing care to ensure good patient outcomes. 


\section{Device for MT}

\subsection{Therapeutic Devices (Recommendation) MT should be provided using an approved device [Grade A].}

\subsubsection{Merci Retriever}

The Merci Retriever (now manufactured by Stryker Corporation) consists of a shape-memory looped wire at the tip that is fed into the occluded area via a microcatheter. The wire loop is then used to ensnare the thrombus.

The MERCI trial was a registry that included 141 patients with acute ischemic stroke who had occlusion of the ICA, MCA, vertebral artery (VA), or BA, had a NIHSS score of $\geq 8$, and were treated within 3-8 hours of onset or were ineligible for IV rt-PA within 3 hours of onset. A recanalization rate (Thrombolysis in Myocardial Infarction [TIMI] grade 2-3) of $48 \%$ was achieved, and $27.7 \%$ of the patients had a good outcome (mRS score 0-2) at 90 days, with a significant difference between those with recanalization $(46.0 \%)$ and those without $(10.4 \%)$. The incidence of symptomatic intracranial hemorrhage (SICH) was $7.8 \% .{ }^{10)}$ Subsequently, the Multi-MERCI trial was conducted also in patients who did not achieve recanalization with IV rt-PA. ${ }^{11)}$ The Japanese regulatory authorities granted approval for the Merci Retriever in April 30, 2010 based on the data of these two clinical studies. Postmarketing surveillance was conducted in all patients treated during the 3 -year period following approval because the device had not been used in Japan at the time of approval. A total of 2,497 patients were registered. The incidence of serious adverse events for which a relationship with the procedure could not be ruled out was $8.4 \%$, and serious adverse events for which a relationship with the device could not be ruled out was $5.2 \%$. These incidences did not differ substantially from the pooled data of the MERCI and MultiMERCI trials, $10.2 \%$ and $3.6 \%$, respectively. ${ }^{10,11)}$ The endpoints in the Japanese postmarketing surveillance were generally good, at $23.8 \%$ for all intracranial hemorrhage (vs. $37.7 \%$ in the pooled data), $7.6 \%$ for SICH (vs. 8.3\%), 73.6\% for recanalization as defined by Thrombolysis in Cerebral Infarction (TICI) grade $\geq 2 \mathrm{a}$ (vs. $64.4 \%$ ), and $19.5 \%$ for mortality at 90 days (vs. $32.4 \%$ ), except that the good outcome (mRS score $0-2)$ rate was inferior to the pooled data $(22.2 \%$ vs. $32.4 \%$ ).

\subsubsection{Penumbra System and Penumbra Aspiration Catheter}

The Penumbra System (Penumbra, Inc.) consists of a relatively large-bore catheter (reperfusion catheter), fed into intracranial arteries, that is connected to a strong aspiration pump and used to aspirate arterial thrombi.

In the Penumbra Pivotal Stroke Trial conducted in the United States, 125 patients presenting within 8 hours of onset with an NIHSS score of $\geq 8$ and large artery occlusion and who were ineligible for or refractory to IV rt-PA therapy were enrolled. A total of $81.6 \%$ of the treated vessels were recanalized to TIMI grade $2-3,25.0 \%$ of the patients had a good outcome (mRS score 0-2) at 90 days, and recanalization tended to be associated with better outcomes. ${ }^{13)}$ Mortality was $32.8 \%$. The incidence of SICH was $11.2 \%$. The Penumbra System was approved in Japan on June 9, 2011 on the basis of these results. As with the Merci Retriever, 3-year all-patient postmarketing surveillance was considered necessary for the Penumbra System because it was approved without having been used in Japan. A total of 3,053 patients were registered, and 3,049 were included in the analyses. The rate of post-procedure recanalization to TICI grade $2 \mathrm{a}-3$ was $82.3 \%$, mortality at 90 days was $17.7 \%, 32.6 \%$ of the patients achieved a good outcome (mRS score 0-2), the incidence of intracranial hemorrhage was $16.9 \%$, and the incidence of SICH was $7.1 \%$.

The MAX Series, with improved suction force and accessibility, became available from June 2013, the 5MAX ACE from October 2014, and the ACE68 from September 2017. Currently, a direct aspiration first-pass technique (ADAPT) ${ }^{29)}$ is primarily used. In A Direct Aspiration first Pass Technique For Acute Stroke Thrombectomy (ADAPT FAST) study, which evaluated the efficacy of ADAPT with the 5MAX and 5MAX ACE, 78\% of the patients achieved recanalization to TICI grade $2 \mathrm{~b}-3,40 \%$ of the patients achieved a good outcome (mRS score 0-2) at 90 days, and SICH did not occur. ${ }^{30)}$

Device improvements are ongoing to address demand for better suction and accessibility with larger bore sizes. The JET7 was released in January 2020, and partial insurance reimbursement for catheter use alone began in 2019.

\subsubsection{Solitaire FR}

A neck-bridge stent (Solitaire AB) intended for use in preventing coil herniation into the parent vessel in coil embolization for wide-neck aneurysms was successfully used to achieve recanalization in acute cerebral artery occlusion, ${ }^{31)}$ and ev3/Covidien (now Medtronic PLC) developed the Solitaire FR in response to this finding. The device, which consists of net-like laser-cut nitinol wrapped onto sheets, is guided to the occluded vessel via a microcatheter. 
The Solitaire FR With the Intention For Thrombectomy (SWIFT) Trial ${ }^{14)}$ was an RCT controlled with the Merci Retriever. The final recanalization rate (TIMI grade $2-3$ ) was $88.9 \%$ in the Solitaire group vs. $67.3 \%$ in the Merci group, and the rate of a good clinical outcome, defined as an mRS score of $0-2$ at 90 days or improvement in the NIHSS score of 10 or more, or maintaining the premorbid mRS score, was $58.2 \%$ vs. $33.3 \%$, and mortality at 90 days was $17.2 \%$ vs. $38.2 \%$, respectively. Each endpoint was significantly better in the Solitaire group. Based on these findings, the Solitaire FR was approved in the United States in March 2012 and in Japan in December 2013, qualifying for insurance reimbursement in July 2014.

The North American Solitaire Stent Retriever Acute Stroke registry (NASA) study ${ }^{32)}$ was conducted as postmarketing surveillance of the Solitaire FR in North America. A total of 354 patients treated with MT using the Solitaire FR at 24 sites were registered. The rate of recanalization to TICI grade 2a-3 was $87.5 \%, 42 \%$ of the patients had good recovery (mRS score $0-2$ ) at 90 days, and $30.2 \%$ of the patients died. The Systematic Evaluation of Patients Treated with Neurothrombectomy Devices for Acute Ischemic Stroke (STRATIS) registry ${ }^{33)}$ was a prospective investigation of patients receiving first-line treatment with the Solitaire or Mindframe Capture LP (unapproved in Japan) in the United States. A total of 984 patients registered at 55 sites were included in the analyses, with the Solitaire used in $96.9 \%$. The rate of recanalization (TICI grade $2 \mathrm{~b}-3$ ) was $87.9 \%$. At 90 days, $56.5 \%$ of the patients achieved a good outcome (mRS score $0-2$ ), and $14.4 \%$ died.

Based on the data of the DAWN trial ${ }^{24)}$ and DEFUSE3 $^{25}$ (section 2.1.3), the device was approved in Japan in November 2019 for the indication of acute ischemic stroke within 24 hours of the time last known well.

\subsubsection{Trevo Pro}

This stent retriever developed by Concentric (now Stryker Corporation) consists of shape-memory, net-like, laser-cut nitinol formed into a tube. This device differs from the Solitaire FR in the thinness of its struts and its delivery wire mounting mechanism. Like the Solitaire, however, the Trevo Pro is guided via a microcatheter to the site of occlusion, where it is deployed to retrieve thrombi.

The efficacy of the Trevo was demonstrated in the Randomized Trial Evaluating Performance of the Trevo Retriever Versus the Merci Retriever in Acute Ischemic Stroke (TREVO2), in which the proportion of patients achieving recanalization of TICI grade $\geq 2 \mathrm{~b}$ was $67.8 \%$ in the Trevo group and
$43.4 \%$ in the Merci group. Although the Trevo group had significantly more patients with a good outcome (mRS score $0-2)$ at 90 days $(40.0 \%$ vs. $21.8 \%$ ), mortality was higher in the Trevo group at $34.1 \%$ than in the Merci group at $24.1 \% .{ }^{15)}$

Based on the TREVO2 data, the Trevo Pro was approved in the United States in August 2012. In Japan, the Trevo ProVue with improved X-ray fluoroscopic visualization was approved in March 2014 and qualified for insurance reimbursement in July 2014. The third-generation Trevo XP ProVue, with an opened end, became available in March 2016.

The postmarketing TREVO Stent-Retriever Acute Stroke (TRACK) registry ${ }^{34)}$ in the United States included 634 patients at 23 sites in whom the Trevo was used as first-line treatment. The median time from onset to presentation was 160 minutes, the median time from presentation to puncture was 118 minutes, and the median time from puncture to recanalization was 67 minutes. The rate of recanalization (TICI grade $2 \mathrm{~b}-3$ ) was $80.3 \%$. At 90 days, $47.9 \%$ of the patients achieved a good outcome (mRS score 0-2), and $19.8 \%$ died.

Based on the data of the DAWN trial ${ }^{24)}$ (section 2.1.3), the device was approved in the United States in February 2018 and in Japan in March 2019 for the indication of acute ischemic stroke within 24 hours of the time last known well. The Trevo Retriever Registry, conducted at 76 sites in 12 countries, included enrollment of 2,008 patients (including about $30 \%$ of whom began treatment 6 or more hours after onset/time last known well). The recanalization rate (mTICI 2b-3) was $92.8 \%$, $55.3 \%$ of the patients achieved a good outcome (mRS score 0-2) at 3 months, the incidence of SICH was $1.7 \%$, and all-cause mortality was $13.9 \%{ }^{35)}$

Currently, the Trevo NXT ProVue, which will feature an improved delivery wire and have superior delivery and guidance with a low-profile catheter, is being developed.

\subsubsection{REVIVE SE}

This stent retriever developed by Codman/Johnson \& Johnson (now Cerenovus/Johnson \& Johnson) has a net-like nitinol basket structure. The device is $4.5 \mathrm{~mm}$ in diameter and $22 \mathrm{~mm}$ in length and is introduced via a microcatheter with a 0.021-inch inside diameter.

The Reperfuse Ischemic Vessels with Endovascular Recanalization device (RIVER)-Japan study (ClinicalTrials.gov Identifier: NCT01895634) was a clinical trial of the REVIVE conducted in Japan from June 2013 to September 2014. In that study, 49 patients with acute ischemic stroke caused by occlusion of the ICA, MCA M1 or M2 segment, BA, or VA were 
enrolled within 8 hours of onset. The recanalization rate of TICI grade $\geq 2$ a was $73.5 \%, 66.7 \%$ of the patients obtained an mRS score of $0-2$ or an improvement in the NIHSS score of $\geq 10$ compared with the baseline score at 90 days, and $6.3 \%$ had $\mathrm{SICH} .{ }^{36)}$ Based on these findings, the device was approved in Japan in January 2016, qualifying for insurance reimbursement in February 2016.

\subsubsection{Tron $F X$}

The Tron FX, a stent retriever developed by the Japanese company Biomedical Solutions, has a nitinol self-expanding stent that is narrowed to decrease the surface area of vessel wall contact and allow the stent to better reach distal vessels. The device is available in two sizes: $4.0-\mathrm{mm}$ diameter with a length of $20 \mathrm{~mm}$ and $2.0-\mathrm{mm}$ diameter with a length of $15 \mathrm{~mm}$. The device is guided via a microcatheter to the site of occlusion, where it deploys by self-enlargement to retrieve thrombi.

A clinical trial of the device in Japan took place from October 2016 to November 2017. In all, 50 patients with acute ischemic stroke caused by occlusion of the ICA, MCA M1/M2/M3 segment, ACA A1/A2 segment, VA, BA, or PCA P1/P2 segment were enrolled within 8 hours of onset. The recanalization rate of TICI grade $\geq 2 \mathrm{a}$ was $80 \%$, the rate of $2 \mathrm{~b}$ or greater was $70 \%, 66.7 \%$ of the patients achieved an mRS score of $0-2$ or an improvement in the NIHSS score of $\geq 10$ compared with the baseline score at 90 days, and $2.0 \%$ had SICH. The device is available in a small diameter size of $2.0 \mathrm{~mm}$. In all, 22 patients with M2/M3 occlusion were enrolled at this size. The rate of TICI $\geq 2 \mathrm{~b}$ was $77.3 \%$, and $85.7 \%$ achieved an mRS score of $0-2$ or an improvement in the NIHSS score of $\geq 10$ compared with the baseline score at 90 days. Based on these findings, the Tron FX was approved in December 2018, qualifying for insurance reimbursement in February 2019.

\subsubsection{EmboTrap}

This stent retriever developed by Neuravi (now Cerenovus/Johnson \& Johnson) has a two-layer construction featuring an outer cage and an inner channel. It captures thrombi differently from other stent retrievers. The device is introduced via a microcatheter with an inside diameter of 0.021 inches or greater.

In the Analysis of Revascularization in Ischemic Stroke with EmboTrap (ARISE II) study in the United States and Europe, the EmboTrap was used without additional therapy. The revascularization rate in target vessels within three passes (mTICI $\geq 2 \mathrm{~b}$ ) was $80.2 \%$, the mTICI $2 \mathrm{c} / 3$ rate was $64.8 \%$, and the
mTICI 3 rate was $43.6 \%$. The revascularization rate in the first pass was $51.8 \%$. The rate of SICH within 24 hours of procedure completion was $5.2 \%, 70.6 \%$ of the patients had a good outcome (mRS score 0-2) at 90 days, and mortality was $9.0 \%{ }^{37)}$

Based on the results of ARISE II, the EmboTrap was approved in the United States in May 2018. In Japan, the device was approved in June 2019 and qualified for insurance reimbursement in August 2019.

\subsubsection{Sofia}

The Sofia (Terumo/MicroVention) was the first aspiration catheter for use in MT to be indicated for ADAPT in Japan. Hybrid braid and coil reinforcement in the catheter shaft and enhanced flexibility of the catheter end enable both excellent distal access and kink resistance in tortuous vessels. The SOFIAFLOW Plus with a 0.070-inch lumen (6 Fr) and SOFIAFLOW with a 0.055-inch lumen (5 Fr) qualified for insurance reimbursement in September 2019 and November 2019, respectively.

\subsubsection{AXS Catalyst, AXS Vecta}

The AXS Catalyst 6 with 0.060-inch lumen, an aspiration catheter developed by Stryker Corporation, offers kink resistance and lesion access with a nitinol and stainless-steel coiled braid. This device was approved in May 2019 and qualified for insurance reimbursement in October 2019. The AXS Catalyst 7 with a 0.068-inch lumen, which has a nitinol coiled braid, became available for use in February 2020. Each catheter has a rounded shaft tip and hydrophilic coating for excellent accessibility. The AXS Vecta aspiration catheter, which was developed by InNeuroCo, Inc. and is marketed outside Japan by Stryker Corporation, contains a nitinol and stainless-steel-coiled braid. Approval for the AXS Vecta71 with a 0.071-inch lumen and AXS Vecta74 with a 0.074-inch lumen is pending in Japan.

\subsubsection{REACT}

The React catheter (Medtronic Plc) is an aspiration catheter that can be guided to intracranial arteries. This device is available as the React 68 with a 0.068-inch lumen and React 71 with a 0.071-inch lumen. The support layer of the catheter uses nitinol, a shape-memory alloy, to achieve both durability and lesion access in tortuous vessels. The React is compatible with the Solitaire, and thrombi can be aspirated and retrieved using the dedicated Riptide aspiration system or manual aspiration with a syringe. The device was approved in Japan in February 2020, and it should become eligible for insurance reimbursement sometime this year. 


\subsubsection{Versi Retriever}

The Versi Retriever, a stent retriever developed by NeuroVasc Technologies, Inc., has a nitinol self-expanding stent with a segmented design that ensures close contact with the vessel wall in tortuous vessels, applies less mechanical stress to vessels during passage, and prevents the scattering of thrombi to distal blood vessels. ${ }^{38,39)}$ The device is available in eight sizes with diameters of $3.0-5.0 \mathrm{~mm}$ and lengths of $10-35 \mathrm{~mm}$. Like other stent retrievers, it is guided via a microcatheter to the site of occlusion, where it deploys by self-enlargement to retrieve thrombi. An investigator-initiated clinical trial began in Japan in January 2019.

\subsection{Conditions for Device Eligibility (Recommendation)}

Medical devices should be used in accordance with their approval conditions.

The devices approved and in use in Japan at the time of the creation of these Guidelines were the stent retrievers Solitaire, Trevo, Tron FX, and EmboTrap and the aspiration catheters Sofia, AXS Catalyst, Penumbra, and REACT. These devices are approved for use in achieving recanalization in patients with acute ischemic stroke within 8 hours of onset for whom IV rt-PA is not indicated or who did not respond to IV rt-PA. Based on the findings of subsequent clinical studies, ${ }^{24,25)}$ the indications for the Trevo retriever and Solitaire were expanded to allow therapy to start within 24 hours of onset.

Since the approval conditions do not necessarily agree with scientific evidence from clinical research on MT, the use of these devices must be carefully weighed in patients with conditions not listed in the package inserts.

Newly approved medical devices should be used in accordance with their approval conditions and specifications.

\section{Eligibility for Treatment}

\section{(Recommendation)}

Scientific evidence supports the effectiveness of MT for acute ischemic stroke caused by large artery occlusion. MT is recommended when the following eligibility conditions are met (see Figure 1).

1) For early acute ischemic stroke, endovascular therapy (MT) with a stent retriever or aspiration catheter within 6 hours of onset in addition to medical therapy that includes IV rt-PA (alteplase) is recommended for patients who meet all of the following: (1) have been diagnosed with an occlusion of a major cerebral artery of the anterior circulation (ICA or MCA M1 segment); (2) have a premorbid mRS score of 0 or 1 ; (3) have an ASPECTS of $\geq 6$ on head CT or MRI DWI; (4) have an NIHSS score of $\geq 6$; and (5) are at least 18 years of age [Grade A].

2) For ischemic stroke appearing to be caused by acute occlusion of the ICA or MCA M1 segment beyond 6 hours since the time last known well, MT within 16 hours of onset or the time last known well is strongly recommended in patients with a premorbid mRS score of 0 or 1 , an NIHSS score of $\geq 10$, and ASPECTS of $\geq 7$ on MRI DWI [Grade A]. In addition, MT within 24 hours of the time last known well is recommended for patients with a mismatch between ischemic core volume (head CT perfusion imaging or MRI DWI) and neurological deficits or a hypoperfusion lesion on perfusion imaging [Grade B].

However, in Japan, it should be noted that the intended use listed in the package insert of all thrombectomy devices other than the Trevo and Solitaire involves "acute ischemic stroke within 8 hours of onset."

3) Although there is insufficient scientific evidence for MT within 6 hours of onset in patients with a large ischemic core having an ASPECTS of $<6$, with mild neurological deficits with an NIHSS score of $<6$, with acute occlusion of the MCA M2 segment or BA, or in patients with a premorbid mRS score of $\geq 2$, MT may be considered if careful consideration of the individual patient's condition shows that the efficacy benefits outweigh the safety risks [Grade C1].

4) Automated image analysis software capable of rapidly measuring ischemic core volume and detecting hypoperfusion lesions can be used for decision-making in MT when perfusion imaging would not be time-consuming [Grade C1].

\subsection{RCTs of MT}

\subsubsection{Controlled Trials of Older-generation Devices}

In February 2013, the results of the Interventional Management of Stroke (IMS) III, ${ }^{16)}$ SYNTHESIS Expansion, ${ }^{17)}$ and Mechanical Retrieval and Recanalization of Stroke Clots Using Embolectomy (MR-RESCUE) ${ }^{18)}$ RCTs of endovascular therapy for acute ischemic stroke were announced.

IMS III ${ }^{16)}$ was an RCT in patients with acute ischemic stroke within 3 hours of onset, and it compared outcomes in a 222-patient group that received IV rt-PA alone with those in a 656-patient group that received additional endovascular therapy including MT. An mRS score of 0-2 at 90 days, which was the primary endpoint, was not significantly different between the groups, at $38.7 \%$ and $40.8 \%(p=0.25)$. The SICH rate was comparable at $5.9 \%$ and $6.2 \%$ 


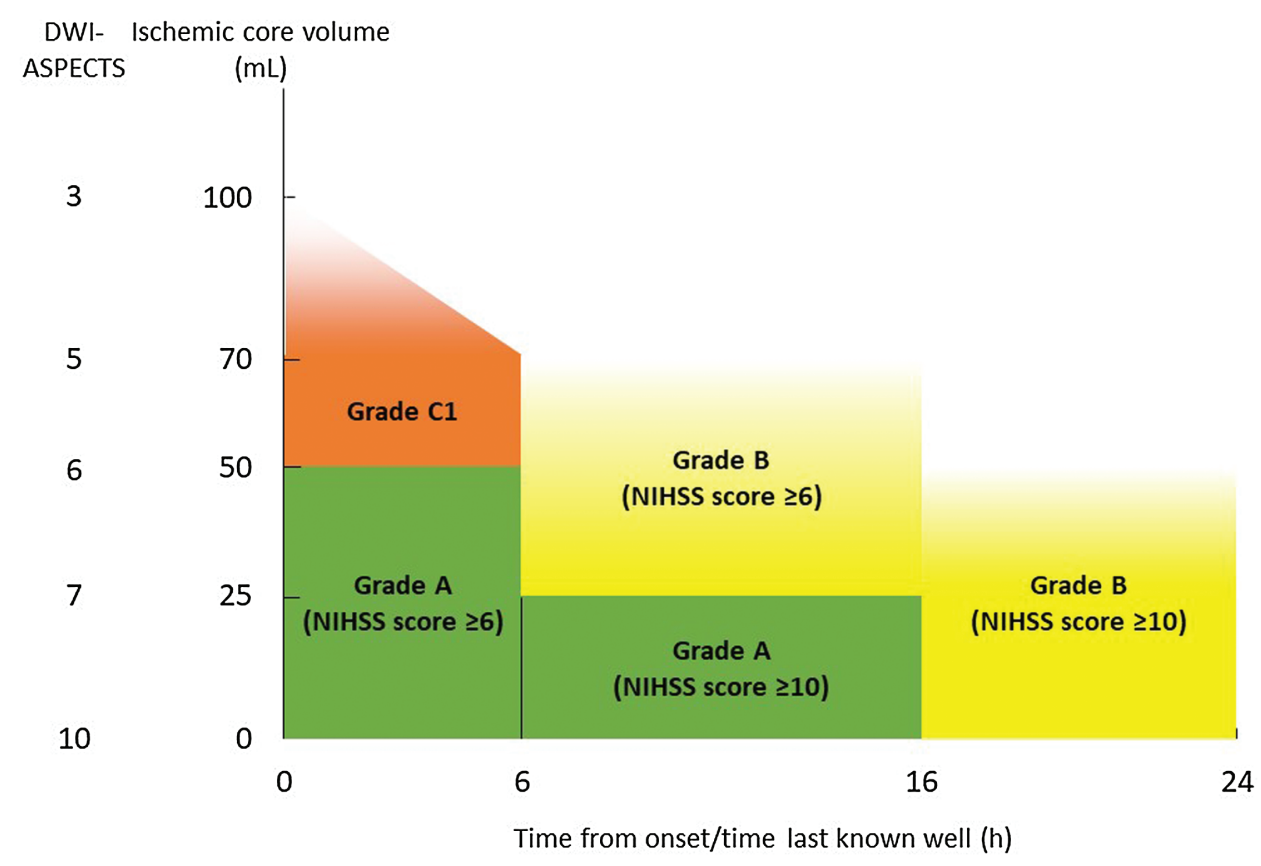

Fig. 1 Recommendation grades for treatment eligibility in patients with ICA or MCA M1 segment occlusion. NIHSS: National Institutes of Health Stroke Scale, DWI-ASPECTS: Alberta Stroke Programme Early CT Score on diffusion-weighted imaging.

$(\mathrm{p}=0.83)$. This study was prematurely terminated before reaching the initial enrollment target of 900 patients because the independent data safety monitoring board found that adding endovascular therapy was not shown to improve patient outcomes.

SYNTHESIS Expansion, ${ }^{17}$ in which patients with acute ischemic stroke within 4.5 hours of onset were randomized to IV rt-PA plus endovascular therapy that included MT, failed to demonstrate the efficacy of endovascular therapy.

MR RESCUE ${ }^{18)}$ was a controlled trial involving 118 patients with acute ischemic stroke due to anterior circulation large artery occlusion within 8 hours of onset who were randomized to endovascular therapy or standard therapy that included IV rt-PA on the basis of penumbra status evaluated on CT or MR perfusion imaging. Endovascular therapy was not shown to be superior, regardless of penumbra status.

Although these three trials failed to demonstrate the superiority of endovascular therapy to medical therapy alone, the results of multiple studies demonstrating the superiority of endovascular therapy were published from 2014 to 2015.

\subsubsection{Controlled Trials in Early Presenting Acute ischemic stroke (Table 1)}

The Multicenter Randomized Clinical Trial of Endovascular Treatment for Acute Ischemic Stroke in the Netherlands (MR CLEAN) ${ }^{20)}$ was an RCT that compared medical therapy including IV rt-PA to endovascular therapy added to medical therapy in patients with acute ischemic stroke caused by large artery occlusion of the anterior circulation within 6 hours of onset. A total of 502 patients with large artery occlusion confirmed by CT angiography (CTA) were randomized to an endovascular therapy group (233 patients) or a medical therapy group (267 patients). Shift analysis of mRS scores at 90 days showed significantly better outcomes in the endovascular therapy group than in the medical therapy group (adjusted common odds ratio [OR]: 1.67; 95\% confidence interval [CI]: 1.21-2.30). Moreover, a significantly higher proportion of patients in the endovascular therapy group had an mRS score of $0-2$ at 90 days (32.6\% vs. $19.1 \%$, OR: 2.05 , 95\% CI: $1.39-3.38$ ). In contrast, no differences were seen in mortality $(21 \%$ vs. $22 \%$ ) or $\mathrm{SICH}(7.7 \%$ vs. $6.4 \%)$. These findings represented the first demonstration of the efficacy of endovascular therapy in acute ischemic stroke. Many RCTs were prematurely terminated following interim analyses. A 2-year follow-up of MR CLEAN ${ }^{40)}$ showed better independence in daily activities in the endovascular therapy group than in the medical therapy group (adjusted common OR [acOR]: 1.68; 95\% CI: 1.15-2.45). Although quality of life was better, mortality was comparable $(26.0 \%$ vs. $31.0 \%)$.

The Endovascular Treatment for Small Core and Anterior Circulation Proximal Occlusion with 


\begin{tabular}{|c|c|c|c|c|c|c|c|c|c|c|c|c|c|}
\hline & \multicolumn{3}{|c|}{ Patient inclusion criteria } & \multirow[b]{2}{*}{$\begin{array}{l}\text { Number } \\
\text { of } \\
\text { patients }\end{array}$} & \multirow[b]{2}{*}{$\begin{array}{l}\text { Treatment } \\
\text { group } \\
\text { recanalization } \\
\text { rate (\%) }\end{array}$} & \multicolumn{3}{|c|}{ mRS score $0-2$ at 90 days (\%) } & \multirow[b]{2}{*}{$\begin{array}{c}\text { mRS shift } \\
\text { acOR } \\
(95 \% \mathrm{CI})\end{array}$} & \multicolumn{2}{|c|}{ Mortality (\%) } & \multicolumn{2}{|c|}{ SICH (\%) } \\
\hline & $\begin{array}{c}\text { Time } \\
\text { Window } \\
\text { (h) }\end{array}$ & $\begin{array}{l}\text { NIHSS } \\
\text { score }\end{array}$ & $\begin{array}{c}\text { Cerebral } \\
\text { parenchyma } \\
\text { diagnostic } \\
\text { imaging }\end{array}$ & & & $\begin{array}{l}\text { Treatment } \\
\text { group }\end{array}$ & $\begin{array}{l}\text { Control } \\
\text { group }\end{array}$ & $\begin{array}{c}\text { Adjusted } \\
\text { OR/RR } \\
(95 \% \mathrm{CI})\end{array}$ & & $\begin{array}{l}\text { Treatment } \\
\text { group }\end{array}$ & $\begin{array}{l}\text { Control } \\
\text { group }\end{array}$ & $\begin{array}{l}\text { Treatment } \\
\text { group }\end{array}$ & $\begin{array}{c}\text { Control } \\
\text { group }\end{array}$ \\
\hline MR CLEAN ${ }^{20)}$ & $\leq 6$ & $\geq 2$ & - & 500 & 58.7 & 32.6 & 19.1 & $\begin{array}{c}2.16 \\
(1.39-3.38)\end{array}$ & $\begin{array}{c}1.67 \\
(1.21-2.3)\end{array}$ & 21 & 22 & 7.7 & 6.4 \\
\hline ESCAPE $^{21)}$ & $\leq 12$ & $\geq 6$ & $\begin{array}{c}\text { ASPECTS } \geq 6 \\
\text { and non-poor } \\
\text { collateral } \\
\text { circulation }\end{array}$ & 316 & 72.4 & 53 & 29.3 & 1.7 & 3.1 & 10.4 & 19 & 3.6 & 2.7 \\
\hline EXTEND-IA ${ }^{22)}$ & $\leq 6$ & - & $\begin{array}{c}\text { Ischemic core } \\
<70 \mathrm{~mL} \text { and } \\
\text { target } \\
\text { mismatch } \\
\text { present }\end{array}$ & 70 & 86 & 71 & 40 & $\begin{array}{c}4.2 \\
(1.2-12)\end{array}$ & $\begin{array}{c}2.1 \\
(1.2-3.8)^{*}\end{array}$ & 9 & 20 & 0 & 6 \\
\hline $\begin{array}{l}\text { SWIFT } \\
\text { PRIME }^{23)}\end{array}$ & $\leq 6$ & $8-9$ & $\begin{array}{c}\text { Ischemic } \\
\text { core }<50 \mathrm{~mL} \\
\text { and target } \\
\text { mismatch }(+) \rightarrow \\
\text { ASPECTS } \geq 6\end{array}$ & 196 & 88 & 60 & 35 & $\begin{array}{c}1.7 \\
(1.23-2.33)\end{array}$ & $\begin{array}{c}2.63 \\
(1.57-4.4)\end{array}$ & 9 & 12 & 0 & 3 \\
\hline REVASCAT $^{33)}$ & $\leq 8$ & $\geq 6$ & $\begin{array}{c}\text { ASPECTS } \geq 7 / \\
\text { DWI- } \\
\text { ASPECTS } \geq 6\end{array}$ & 206 & 65.7 & 43.7 & 28.2 & $\begin{array}{c}2.1 \\
(1.1-4.0)\end{array}$ & $\begin{array}{c}1.7 \\
(1.05-2.8)\end{array}$ & 18.4 & 15.5 & 1.9 & 1.9 \\
\hline THERAPY ${ }^{35)}$ & NR & $\geq 8$ & $\begin{array}{l}\leq 1 / 3 \text { of middle } \\
\text { cerebral artery } \\
\text { region }\end{array}$ & 108 & 73 & 38 & 30 & $\begin{array}{c}1.4 \\
(0.6-3.3)\end{array}$ & $\begin{array}{c}1.76 \\
(0.86- \\
3.6)^{*}\end{array}$ & 12 & 24 & 9.3 & 9.7 \\
\hline THRACE ${ }^{36)}$ & $\leq 5$ & $10-25$ & - & 414 & 68.8 & 53 & 42 & $\begin{array}{c}1.55 \\
(1.05-2.30)\end{array}$ & $\begin{array}{c}1.39 \\
(0.99- \\
1.97)\end{array}$ & 12 & 13 & 2 & 2 \\
\hline PISTE $^{37)}$ & $\leq 6$ & - & $\begin{array}{c}\leq 1 / 3 \text { of middle } \\
\text { cerebral artery } \\
\text { region }\end{array}$ & 65 & 87 & 51 & 40 & $\begin{array}{c}2.12 \\
(0.65-6.94)\end{array}$ & $\begin{array}{c}2.59 \\
(0.93- \\
7.24)\end{array}$ & 21.2 & 12.5 & 0 & 0 \\
\hline
\end{tabular}

*Unadjusted OR. Recanalization rate: Thrombolysis in Cerebral Infarction (TICI) grade 2b-3, acOR: adjusted common odds ratio, NR: not reported, OR: odds ratio, RR: rate ratio (ESCAPE) or risk ratio (SWIFT PRIME), SICH: symptomatic intracranial hemorrhage. 
Emphasis on Minimizing CT to Recanalization Times (ESCAPE) $^{21)}$ trial was an RCT with a group that received medical therapy including IV rt-PA and a group that received endovascular therapy with a stent retriever in patients with acute ischemic stroke within 12 hours of onset, an ASPECTS of $\geq 6$, occlusion of the ICA or MCA M1 or large M2 segment confirmed on CTA, and moderate-to-good collateral circulation. The endovascular therapy group had significantly better mRS scores at 90 days (acOR: 3.1; 95\% CI: 2.0-4.7), more patients with better outcomes (mRS score of $0-2)(53.0 \%$ vs. $29.3 \%$; OR, 1.7), and fewer deaths ( $10 \%$ vs. $19 \%$; OR, 0.5). SICH did not differ between the groups (3.6\% vs. $2.7 \%)$.

The Extending the Time for Thrombolysis in Emergency Neurological Deficits-Intra-Arterial (EXTEND-IA) ${ }^{22}$ trial was an RCT that compared IV rt-PA to added endovascular therapy with MT using the Solitaire FR in patients with acute ischemic stroke within 4.5 hours of onset who had occlusion of the ICA or MCA (M1 or M2 segment) confirmed by CTA, an ischemic penumbra area on CT perfusion (CTP) imaging (i.e., "target mismatch"), and an ischemic core volume of less than $70 \mathrm{~mL}$. Reperfusion volume at 24 hours (decrease in area with Tmax $\geq 6$ s) was significantly higher in the endovascular therapy group (median 100\% vs. $37 \%$, p <0.001; adjusted OR [aOR]: 4.7 , and significantly more patients in that group experienced an improvement in early neurological deficits (as defined by an NIHSS score improvement of $\geq 8$ or a score of 0 or 1 three days after onset) ( $80 \%$ vs. $37 \%, p=0.002$; aOR, 6.0 ). The proportions of patients with an mRS score of 0-2 at 90 days were $40 \%$ in the IV rt-PA group and $71 \%$ in the endovascular group ( $p=0.01$; aOR, 4.2). SICH and mortality did not differ between the groups.

The Solitaire FR With the Intention For Thrombectomy as PRIMary Endovascular Treatment for Acute Ischemic Stroke (SWIFT PRIME) ${ }^{23)}$ trial was an RCT in patients with acute ischemic stroke caused by occlusion of the intracranial ICA or MCA M1 segment who underwent IV rt-PA within 4.5 hours of onset and were able to start MT within 6 hours of onset. The inclusion criteria initially required the detection of "target mismatch" on CTP or MR perfusion imaging, but the protocol was later changed to exclude patients with an ASPECTS of $\leq 5$. The endovascular therapy group had significantly better mRS scores at 90 days than the IV rt-PA group ( $p<0.0001$ ) and had a higher proportion of patients with an mRS score of $0-2$ (60\% vs. $35 \%$; risk ratio, 1.70; 95\% CI: 1.23-2.33). SICH and mortality did not differ between the groups.

The Randomized Trial of Revascularization with Solitaire FR Device versus Best Medical Therapy in the Treatment of Acute Stroke Due to Anterior Circulation Large Vessel Occlusion Presenting within Eight Hours of Symptom Onset (REVASCAT) ${ }^{41)}$ was an RCT that compared medical therapy including IV rt-PA with endovascular therapy with the Solitaire FR in patients with acute ischemic stroke within 8 hours of onset having an NIHSS score of $\geq 6$, ASPECTS of $\geq 7$, or DWI-ASPECTS score of $\geq 6$ and occlusion of the intracranial ICA or MCA M1 segment confirmed by CTA or MRA. The mRS scores at 90 days were significantly better in the endovascular therapy group than in the medical therapy group (aOR: 1.7; 95\% CI: 1.05-2.8). SICH occurred in $1.9 \%$ of the patients in each group. Mortality was $18.4 \%$ vs. $15.5 \%$.

The Highly Effective Reperfusion evaluated in Multiple Endovascular Stroke Trials (HERMES) Collaboration conducted a meta-analysis of the patient data from the following five trials: MR CLEAN, ESCAPE, REVASCAT, SWIFT PRIME, and EXTEND-IA. ${ }^{42)}$ The primary endpoint of the mRS score at 90 days was significantly shifted in favor of the endovascular therapy group (acOR: 2.49; 95\% CI: 1.76-3.53), the number needed to treat (NNT) to improve the mRS score by at least 1 point in one patient was 2.6 , and a significantly higher proportion of patients in the endovascular therapy group had an mRS score of $0-2$ at 90 days $(46.0 \%$ vs. $26.5 \%$; aOR: 2.71 ; $95 \%$ CI: $2.07-3.55$ ). No significant difference was seen in mortality at 90 days (15.3\% vs. $18.9 \%$; aOR: 0.73 ; 95\% CI: $0.41-1.13$ ) or SICH within 5 days $(4.4 \%$ vs. $4.3 \%$; aOR: 1.07 ; 95\% CI: 0.62-1.84). Subgroup analysis based on whether IV rt-PA was used showed no significant difference in the effect of endovascular therapy ( $P$ interaction $=0.43$ ), and the acOR was not significant for ASPECTS $\leq 5$, NIHSS score $\leq 10$, or M2 segment occlusion. However, endovascular therapy was found to have a significant effect in those at least 80 years of age (common OR: 3.68), in those randomized 300 or more minutes after onset (common OR: 1.76), and in those for whom IV rt-PA was not indicated (common OR: 2.43). Mortality at 90 days in those at least 80 years of age was lower in the endovascular therapy group (28\% vs. $45 \%$; aOR: $0.60 ; 95 \%$ CI: 0.36-0.99).

THRombectomie des Artères CErebrales (THRACE) ${ }^{43)}$ was a study that compared IV rt-PA alone to added endovascular therapy in patients with occlusion of the ICA, MCA M1 segment, or BA who received IV rt-PA within 4 hours of onset. Significantly more patients in the endovascular therapy group achieved the primary endpoint of an mRS score of $0-2$ at 90 days $(42 \%$ vs. $53 \%$; OR, 1.55 ; 95\% CI: $1.05-2.30$; $\mathrm{p}=0.028)$. 
The Randomized, Concurrent Controlled Trial to Assess the Penumbra System's Safety and Effectiveness in the Treatment of Acute Stroke (THERAPY) ${ }^{44)}$ was an RCT that compared thrombus aspiration with the Penumbra System plus IV rt-PA to IV rt-PA alone in patients with occlusion of the ICA or MCA who received IV rt-PA within 4.5 hours of onset. One inclusion criterion of the study required a thrombus length of more than $8 \mathrm{~mm}$. The proportion of patients with an mRS score of 0-2 at 90 days tended to be higher in the endovascular therapy group, but it was not significant on intention-to-treat (ITT) analysis (38\% vs. $30 \%$; OR: $1.4 ; 95 \%$ CI: $0.60-3.3 ; \mathrm{p}=0.44$ ). Ordinal per protocol analysis showed a significant improvement in the mRS score, which was a secondary endpoint (OR: 2.2; 95\% CI: 1.0-5.0; $\mathrm{p}=0.047$ ).

The Pragmatic Ischaemic Stroke Thrombectomy Evaluation (PISTE) ${ }^{45}$ ) was an RCT that compared IV rt-PA alone to IV rt-PA and endovascular therapy in patients with occlusion of the intracranial ICA or MCA M1 or M2 segment who were able to undergo IV rt-PA within 4.5 hours of onset. In this trial, the investigators selected an approved device to use. The proportion of patients with an mRS score of 0-2 at 90 days, which was the primary endpoint, was not significantly different on ITT analysis (aOR: 2.12; 95\% CI: 0.65-6.94; $\mathrm{p}=0.20$ ). However, per protocol analysis showed endovascular therapy to be effective (aOR: 4.9; 95\% CI: 1.2-19.7; $\mathrm{p}=0.02$ ).

\subsubsection{Controlled trials in late presenting acute ischemic stroke}

The DWI or CTP Assessment with Clinical Mismatch in the Triage of Wake-Up and Late Presenting Strokes Undergoing Neurointervention with Trevo (DAWN) trial $^{24)}$ was an RCT that compared endovascular therapy with the Trevo (Stryker Corporation) plus medical therapy to medical therapy alone in patients with a clinical imaging mismatch (CIM) between neurological deficits and ischemic core volume in acute ischemic stroke caused by occlusion of the ICA or MCA M1 segment 6-24 hours after the time last known well, including cases in which the time of onset was unknown. The ischemic core volume was measured using RAPID (iSchemaView, Inc.) on DWI or cerebral blood flow imaging of CTP. A CIM was defined as an NIHSS score of $\geq 10$ plus ischemic core of $<21 \mathrm{~mL}$ in those at least 80 years of age and an NIHSS score of $\geq 10$ plus ischemic core of $<31 \mathrm{~mL}$ or NIHSS score of $\geq 20$ plus ischemic core of $<51 \mathrm{~mL}$ in those younger than 80 years. The initial recruitment target was 500 patients, but the study was terminated early following a recommendation by the independent safety monitoring committee based on an interim analysis conducted after 206 patients had been enrolled. A total of 107 patients were randomized to the endovascular therapy group and 99 to the medical therapy group. The primary endpoint, which was the utilityweighted mRS score at 3 months, was significantly better in the endovascular therapy group, and the proportions of patients with an mRS score of $0-2$ were markedly different, at $48 \%$ vs. $13 \%$. No significant differences were seen in SICH (6\% vs. $3 \%$ ) or mortality (19\% vs. $18 \%)$. The recanalization rate at 24 hours was $77 \%$ in the endovascular therapy group and $36 \%$ in the medical therapy group. The mean time from the time last known well to randomization was 13 hours in each group, but the likelihood of a better outcome from earlier treatment was higher in the endovascular therapy group.

The Endovascular Therapy Following Imaging Evaluation for Ischemic Stroke (DEFUSE3) trial ${ }^{46)}$ was an RCT that compared endovascular therapy plus medical therapy to medical therapy alone in patients with acute ischemic stroke caused by occlusion of the ICA or MCA M1 segment 6-16 hours after the time last known well who were found to have a target mismatch (ischemic core $<70$ $\mathrm{mL}$ and mismatch ratio $>1.8$ between the ischemic core and the hypoperfusion lesion) according to RAPID software (iSchemaView, Inc.). The initial recruitment target was 500 patients, but the trial was terminated early based on significant results achieved at the time when 182 patients had been enrolled. The mRS scores at 90 days were significantly shifted in favor of the endovascular therapy group (OR: 2.77; $\mathrm{p}<0.001$ ). The endovascular therapy group tended to have lower mortality (14\% vs. $26 \%$; $\mathrm{p}=0.05$ ), but SICH occurrence did not differ between the groups ( $7 \%$ vs. $4 \% ; p=0.75)$. In the endovascular therapy group, $76 \%$ of the patients achieved reperfusion of TICI $\geq 2 \mathrm{~b}$.

\subsection{Eligibility Based on Diagnostic Imaging and Neurological Deficits}

\subsubsection{Eligibility Based on Cerebrovascular Evaluation}

All RCTs that showed superior efficacy for endovascular therapy compared with medical therapy included patients diagnosed with large artery occlusion of the anterior circulation (ICA or MCA M1 segment) confirmed on CTA- or MRA-based cerebrovascular assessment. Because the efficacy of endovascular therapy was not demonstrated in IMS III and SYNTHESIS Expansion, which did not require cerebrovascular assessment, non-invasive cerebrovascular assessment with initial diagnostic imaging is a requirement for assessing eligibility for MT [Grade A]. On the other hand, excessive time spent 
on cerebrovascular assessment and delayed MT treatment would decrease the improvement in outcome. Sites where MT is performed should organize a system that enables cerebrovascular assessment without delay [Grade A].

\subsubsection{Eligibility Based on Ischemic Core Volume and Areas of Hypoperfusion}

CT or MRI assessment of the cerebral parenchyma is needed to exclude hemorrhagic stroke and identify/predict the range of ischemia. Early ischemic signs on non-contrast CT (NCCT), areas of low cerebral blood flow on CTP, and areas with a low apparent diffusion coefficient (ADC) on MRI DWI are considered to indicate areas of irreversible ischemia, that is, ischemic cores. RAPID calculates an ischemic core as an area of cerebral blood flow less than $30 \%$ of the contralateral blood flow on CTP or ADC less than $620 \times 10^{-6} \mathrm{~mm}^{2} / \mathrm{s}$ on DWI. A large ischemic core increases the possibility of a poor outcome following MT recanalization or increased intracranial hemorrhage.

Criteria for a "large ischemic core" include involvement of $\geq 33 \%$ of an MCA territory, which is reported to have poor outcomes and higher incidence of SICH in IV rt-PA, ${ }^{47,48}$ infarct volume of at least $100 \mathrm{~mL}{ }^{49)}$ ischemic core volume of at least $54 \mathrm{~mL}$ on CTP, ${ }^{50)}$ and infarct volume of at least 70 $\mathrm{mL}$ on DWI. ${ }^{51)}$ In SWIFT and TREVO2, which compared stent retrievers and the Merci Retriever, patients with infarcted area of one MCA territory $\geq 33 \%$ or infarct volume over $100 \mathrm{~mL}$ were excluded.

Recent RCTs other than MR CLEAN and THRACE used ischemic core assessment based on diagnostic imaging to select patients. Inclusion criteria were ASPECTS of $\geq 6$ by NCCT in ESCAPE, a value of $<70 \mathrm{~mL}$ on CTP in EXTEND-IA, initially a value of $<50 \mathrm{~mL}$ on CTP or DWI but then ASPECTS of $\geq 6$ by NCCT or DWI in SWIFT PRIME, ASPECTS of $\geq 7$ or DWI ASPECTS of $\geq 6$ in REVASCAT, and infarcted area of one MCA territory $<33 \%$ in THERAPY and PISTE. Given the ischemic core assessments common across these RCTs, starting MT within 6 hours of onset is strongly recommended in patients with occlusion of the ICA or MCA M1 segment who have ASPECTS of $\geq 6$ on NCCT or DWI [Grade A].

In DAWN and DEFUSE3, patients presenting late after last known well required ischemic core volume analysis using appropriate analytical software with CTP or DWI; DAWN with the presence of a mismatch with the neurological deficits and DEFUSE3 with a mismatch of hypoperfusion lesions as patient selection criteria. The median (quartiles) ischemic core volume of the patients randomized to the endovascular therapy group was $7.6 \mathrm{~mL}(2-18 \mathrm{~mL})$ in DAWN and 9.4 $\mathrm{mL}(2.3-25.6 \mathrm{~mL})$ in DEFUSE3. Therefore, MT is strongly recommended for patients with occlusion of the ICA or MCA M1 segment 6-16 hours after last known well if the NIHSS score is $\geq 10$ and ischemic core volume is $\leq 25 \mathrm{~mL}$ (which corresponds to DWI-ASPECTS of $\geq 7$ ) [Grade A]. DAWN included patients within 24 hours of onset assessed using the above CIM criteria, and DEFUSE3 included patients with an NIHSS score of $\geq 6$ and ischemic core volume of $<70 \mathrm{~mL}$ having a mismatch with perfusion imaging. MT is recommended when these criteria are fulfilled [Grade B]. However, in Japan, it should be noted that the package insert of all thrombectomy devices other than the Trevo Retriever and Solitaire in Japan calls for use in acute ischemic stroke within 8 hours of onset.

The installation of RAPID or other similar software programs that can rapidly measure ischemic core volume would inform decision-making for MT eligibility. There is a correlation in ischemic core assessment: DWI-ASPECTS of $\geq 7, \geq 5$, and $\geq 3$ points correspond to ischemic core volumes of $\leq 25, \leq 70$, and $\leq 100 \mathrm{~mL}$, respectively. ${ }^{52-56)}$ Clinical-ASPECTS mismatch is reported to approximate the DAWN CIM criteria. ${ }^{57)}$

Areas with delayed perfusion on perfusion imaging are used to predict ischemic penumbra areas that could result in infarction if no reperfusion of the occluded vessel is achieved. In RAPID, areas of hypoperfusion are defined as Tmax $>6$ s. SWIFTPRIME (initially) and DEFUSE3 required a hypoperfusion area to ischemic core volume ratio (mismatch ratio) of at least 1.8 as a patient selection criterion, and EXTEND-IA required a ratio of at least 1.2. ESCAPE used a CTA-based collateral circulation assessment as a substitute for brain tissue perfusion status. In the RCTs, perfusion imaging was used for patient selection in SWIFT PRIME (initially) and EXTEND-IA for patients in early acute ischemic stroke, and in DEFUSE3 for late-onset presentation. When advanced imaging does not delay the treatment, rapid evaluation of ischemic core volume and hypoperfusion lesions using automated software is beneficial for evaluating the efficacy and safety of treatment and can be used in decision-making for MT eligibility [Grade C1].

\subsection{Patients in Whom the Efficacy Is Not Established}

A secondary analysis of HERMES found that the adjusted common odds ratios were not significant for ASPECTS $\leq 5$, NIHSS scores $\leq 10$, and M2 segment occlusion. ${ }^{42)}$ No RCT in BA occlusion has shown MT to be effective. However, there are studies suggesting that MT is effective in patients with 
acute ischemic stroke caused by large artery occlusion featuring a large ischemic core, patients with mild neurological deficits, patients with an MCA M2 segment occlusion, and patients with a BA occlusion. Although sufficient scientific evidence for treatment in these patients is lacking, MT may be considered after careful consideration when the efficacy benefits outweigh the safety risks, mainly within 6 hours of onset [Grade C1].

\subsubsection{Patients with a Large Ischemic Core}

In a matched case-control study of patients who did and did not undergo MT with acute ischemic stroke caused by occlusion of the ICA or MCA M1 or M2 segment and had an ischemic core volume on CTP of $\geq 50 \mathrm{~mL}$ and a $\geq 40 \mathrm{~mL}$ mismatch area that included an eloquent area, MT improved the outcome at 3 months (shift analysis of mRS scores at 90 days, common OR 2.56: 95\% CI: 2.50-8.47, $\mathrm{p}=0.04 ; \mathrm{mRS}$ score of $0-2,23 \%$ vs. $0 \%) .{ }^{58)}$ The HERMES meta-analysis of seven trials in patients with early large artery occlusion of the anterior circulation showed a higher incidence of SICH for MT among patients with ASPECTS of 3-5 (15\% vs. $3 \%, p=0.01$ ), but the acOR of mRS score at 90 days was 2.00 (95\% CI: 1.16-3.46), showing significantly better improvement. ${ }^{59)}$ The images of the patients enrolled in these seven studies were reanalyzed with RAPID to investigate the relationship between ischemic core and penumbra (mismatch) volumes and outcomes. From this analysis, MT offered better outcomes at 90 days than medical therapy in patients with an ischemic core volume of 100-150 mL when effective reperfusion was achieved by MT. ${ }^{60)}$ In the Recovery by Endovascular Salvage for Cerebral Ultra-acute Embolism (RESCUE) Japan Registry 2, a multicenter, prospective, observational study of emergent large vessel occlusion conducted in Japan after the advent of stent retrievers, endovascular therapy produced significantly better outcomes than medical therapy in 504 patients with ICA or M1 segment occlusion and ASPECTS of $\leq 5$ (mRS score after 90 days $\leq 2: 19.8 \%$ vs. $4.2 \%$, aOR: 2.33, 95\% CI: $1.10-4.94$, mRS score after 90 days < 3: $34.9 \%$ vs. $7.5 \%$, aOR: 3.82 , $95 \%$ CI: $2.04-7.18$ ). Moreover, mortality tended to be lower (aOR: 0.59, 95\% CI: 0.32-1.08), and SICH incidence was not increased (aOR: 0.50, 95\% CI: 0.14-1.73). ${ }^{61)}$

\subsubsection{Patients with mild neurological deficits}

Of 32 patients with large artery occlusion having an NIHSS score on admission of $<6$, the 22 who underwent MT had a significantly better improvement in the NIHSS score at discharge than the 10 who did not $(-2.5$ vs. $0 ; \mathrm{p}<0.01){ }^{62)}$ However, in an observational study of 251 patients with an NIHSS score of $<6$ and occlusion of the ICA or M1 or M2 segment (113 of whom underwent endovascular therapy and 138 of whom underwent medical therapy) at 16 medical institutions in North America, Europe, and Asia, no significant intergroup differences were observed in clinical outcomes following multivariate adjustment, and asymptomatic ICH was higher in the endovascular therapy group (aOR: 11.07, 95\% CI: 1.31-93.53). A meta-analysis of four studies that included this study showed no significant differences in clinical outcomes or SICH incidence. ${ }^{63)}$

\subsubsection{Patients with MCA M2 Segment Occlusion}

In 522 patients with M2 segment occlusion within 8 hours of onset in a registry of 10 sites in the United States, MT-treated patients (288) were compared with medically treated patients (234). Those undergoing MT had significantly better outcomes even after adjustment for baseline characteristics (OR: 3.2 ; 95\% CI: $2-5.2 ; \mathrm{p}<0.001){ }^{64)}$ The HERMES meta-analysis of seven studies included 130 patients with M2 segment occlusion (67 in the endovascular therapy group and 63 in the control group). ${ }^{65)}$ The endovascular therapy group had a recanalization rate (mTICI $\geq 2 \mathrm{~b}$ ) of $59.2 \%$. The proportion of patients with a good outcome at 3 months (mRS score $0-2$ ) was $58.2 \%$ vs. $39.7 \%$ (aOR: 2.39, 95\% CI: 1.08-5.28), and the acOR for mRS score improvement was 1.77 (95\% CI: 0.94-3.36). Outcomes were better in the endovascular therapy group, although shift analysis did not show a significant difference. MT produced significantly superior efficacy to medical therapy in patients with an occlusion of the proximal M2 segment (116 patients, aOR: 2.68, 95\% CI: 1.13-6.37), dominant M2 segment (73 patients, aOR: 4.08, 95\% CI: 1.08-15.48), and single M2 segment (123 patients, aOR 2.73, 95\% CI: 1.19-6.27). Post hoc analysis of the RESCUE Japan Registry ${ }^{66)}$ that included 372 patients with M2 segment occlusion (184 in the endovascular therapy group and 188 in the medical therapy group) showed significantly better outcomes in the endovascular therapy group (mRS score of 0-2 after 90 days: OR: $2.09,95 \%$ CI: $1.26-3.47$, death: OR: 0.27 , 95\% CI: 0.08-0.93).

\subsubsection{Patients with BA Occlusion}

A meta-analysis of 17 case series involving MT in patients with BA occlusion ${ }^{67)}$ found a recanalization rate of TICI $\geq 2 \mathrm{~b}$ of $80 \%$, a good outcome at 3 months (mRS score of $0-2$ ) in $43 \%$ of the patients, mortality of $29 \%$, and SICH incidence of $7 \%$. The EVT for Acute Basilar Artery Occlusion Study (BASILAR) was a prospective registry study involving 829 patients 
with BA occlusion within 24 hours of onset. In this study, 647 patients received endovascular therapy, whereas 182 received medical therapy. The endovascular therapy group had significantly better mRS scores at 90 days (acOR: 3.08, 95\% CI: 2.09-4.55) and more patients with mRS scores of 0-3 (aOR: 4.70, 95\% CI: 2.53-8.75), as well as a lower mortality rate (aOR 2.93, 95\% CI: 1.95-4.40). ${ }^{68)}$ The Basilar Artery Occlusion Endovascular Intervention versus Standard Medical Treatment (BEST) ${ }^{69)}$ trial was an RCT that compared endovascular therapy and medical therapy in BA occlusion within 8 hours of onset. In all, 28 sites in China participated in this trial. New enrollment was stopped when 131 patients had been enrolled (66 in the endovascular therapy group and 65 in the medical therapy group, or $38 \%$ of the initial target of 344 patients) because of a high crossover rate $(22 \%$ of the patients in the medical treatment group underwent endovascular therapy) and slow recruitment. The primary endpoint of the mRS score at 90 days of $0-3$ (per ITT analysis) was not significantly different, at $42 \%$ in the endovascular therapy group and $32 \%$ in the medical therapy group (aOR 1.74, 95\% CI: 0.813.74 ), but better outcomes were seen in the endovascular therapy group in the per-protocol analysis (aOR: 2.90, 95\% CI: $1.20-7.03)$ and the as-treated analysis (aOR: 3.02, 95\% CI: 1.31-7.00). SICH incidence tended to be higher in the endovascular therapy group ( $8 \%$ vs. $0 \%, \mathrm{p}=0.06)$, but mortality did not differ $(33 \%$ vs. $38 \%$, aOR: $0.80,95 \%$ CI: $0.37-1.64)$.

\section{Considerations for Treatment}

\subsection{Use with Intravenous Thrombolytic Therapy (Recommendation) IV rt-PA should be given priority in eligible patients [Grade A].}

IV rt-PA is the treatment with the highest level of evidence in acute ischemic stroke. In the National Institute of Neurological Disorders and Stroke (NINDS) rt-PA Stroke Study, ${ }^{2)}$ patients receiving intravenous therapy with rt-PA (alteplase $0.9 \mathrm{mg} / \mathrm{kg}$ ) for acute ischemic stroke within 3 hours of onset had significantly better independence of activities at 3 months compared with the placebo group, whereas mortality did not differ. The European Cooperative Acute Stroke Study (ECASS) III showed IV rt-PA to be effective and safe in patients with acute ischemic stroke treated within 3-4.5 hours of onset. ${ }^{6)} \mathrm{A}$ meta-analysis of this and many other large-scale clinical studies demonstrated that IV rt-PA given within 4.5 hours of onset improves outcomes. ${ }^{70)}$

Similar efficacy was seen in the Japan Alteplase Clinical Trial (J-ACT), ${ }^{71)}$ a prospective single-arm trial with a reduced dose of $0.6 \mathrm{mg} / \mathrm{kg}$ of alteplase conducted in Japan. This treatment became eligible for insurance reimbursement in 2005, and the indication was expanded to within 4.5 hours of onset in 2012. IV rt-PA significantly increases SICH incidence and should therefore be administered in compliance with the third edition of the appropriate use guidelines created by the JSS. ${ }^{26)}$

In HERMES, $83 \%$ of the patients in the thrombectomy group and $87 \%$ in the medical therapy group received IV rt-PA. The efficacy of MT and mortality did not differ according to whether IV rt-PA was administered. A meta-analysis of 13 studies conducted to investigate the relationship between pre-MT IV rt-PA use and treatment outcomes found that more patients who received IV rt-PA than those who did not had a good outcome at 3 months (mRS score of 0-2) (OR: 1.27, 95\% CI: 1.05-1.55, $\mathrm{p}=0.02$ ), fewer deaths (OR: 0.71, 95\% CI: $0.55-0.91, \mathrm{p}=0.006$ ), and a higher recanalization rate (OR: 1.46, 95\% CI: 1.09-1.96, $\mathrm{p}=0.01$ ), and SICH incidence did not increase (OR: 1.11, 95\% CI: $0.69-1.77, \mathrm{p}=0.67) .{ }^{72)}$ The SKIP study in patients with ICA or M1 segment occlusion within 4.5 hours of onset conducted in Japan ${ }^{73)}$ showed a comparable level of good outcomes for thrombectomy alone compared with thrombectomy combined with IV rt-PA, but non-inferiority was not demonstrated. SICH incidence did not differ between the groups, but the incidence of all intracranial hemorrhage, including asymptomatic, was significantly lower in the thrombectomy-only group than in the thrombectomy plus IV rt-PA group (Suzuki et al. presented at International Stroke Conference 2020).

In the Extending the time for Thrombolysis in Emergency Neurological Deficits-Intra-Arterial using Tenecteplase (EXTEND-IA TNK) ${ }^{74)}$ study, tenecteplase (unapproved in Japan) at $0.25 \mathrm{mg} / \mathrm{kg}$ and alteplase at $0.9 \mathrm{mg} / \mathrm{kg}$ were compared as intravenous thrombolytic therapy before MT. The tenecteplase group had a higher recanalization rate (TICI $2 \mathrm{~b} / 3$ ) on initial angiography ( $22 \%$ vs. $10 \%$, non-inferiority $\mathrm{p}=0.002$, superiority $\mathrm{p}=0.03$ ) and better $\mathrm{mRS}$ scores at 90 days (adjusted common OR: 1.7, 95\% CI: 1.0-2.8, $\mathrm{p}=0.04$ ). The EXTEND-IA TNK part II study, which compared doses of tenecteplase, evaluated reperfusion of at least $50 \%$ of vascular occlusion following treatment with tenecteplase $0.4 \mathrm{mg} / \mathrm{kg}$ and tenecteplase $0.25 \mathrm{mg} / \mathrm{kg}$ before MT. There was no betweengroup difference $(19.3 \%$ vs. $19.3 \%$, aRR: $1.03,95 \%$ CI: $0.66-1.61, \mathrm{p}=0.89){ }^{75)}$

In conclusion, endovascular therapy using an intracranial thrombectomy device without using an unapproved rt-PA agent or IV rt-PA for patients indicated for IV rt-PA had no established efficacy or 
safety profile as of the time of these Guidelines and must therefore be avoided in non-research settings.

The Efficacy and Safety of MRI-Based Thrombolysis in Wake-Up Stroke (WAKE-UP) ${ }^{76)}$ study evaluated the efficacy of IV rt-PA versus placebo in patients with stroke on waking or of an unknown time of onset whose DWI ischemic change was unclear on FLAIR (i.e., DWI/FLAIR mismatch). In patients who began treatment within 4.5 hours of symptom recognition and within 1 hour of MRI, more patients in the IV rt-PA group than the placebo group had an mRS score of $0-1$ at 90 days ( $53.8 \%$ vs. $41.8 \%$, aOR: $1.61,95 \%$ CI: 1.09-2.36). On mRS score shift analysis, the adjusted common OR was 1.62 (95\% CI: 1.17-2.23), indicating a significantly better outcome. On the basis of these findings, the 3rd Edition of the Guidelines for Intravenous Thrombolysis (rt-PA) ${ }^{26)}$ states that, when the time of onset is unknown, administering IV rt-PA may be considered within 4.5 hours of symptom recognition if a DWI/FLAIR mismatch is present. However, the efficacy and safety profiles of IV rt-PA followed by thrombectomy have not been established in such patients who have large artery occlusion [Grade C1].

\subsection{Times from Onset to Arterial Puncture and Reperfusion \\ (Recommendation)}

Better outcomes are expected when rapid recanalization of the occluded vessel is associated with MT. When MT is selected, treatment must be started without delay, and recanalization must be achieved as quickly as possible with the optimal approach [Grade A]. When IV rt-PA has been administered, the start of MT must not be delayed for the evaluation of thrombolysis effectiveness [Grade D].

Performing MT as soon as possible in patients whose large artery occlusion status is clear increases the clinical benefit. In a secondary analysis of the relationship of the time from onset to the start of MT (artery puncture) with outcome in HERMES, ${ }^{77}$ ) the common OR of a better mRS score at 90 days compared with medical therapy was 2.79 when the time from onset was 3 hours (95\% CI: 1.96-3.98), 1.98 when the time was 6 hours (1.30-3.00), and 1.57 when the time was 8 hours (0.86-2.88). MT efficacy decreased the later treatment began. The analysis also showed that, in the 390 patients achieving effective reperfusion with MT, a 1-hour delay of reperfusion reduced the proportion of patients with a good outcome at 3 months by 19\% (OR: 0.81, 95\% CI: 0.71-0.92). Reasonable targets for treatment times that have been advocated include no more than 50 minutes from diagnostic imaging to artery puncture, no more than 75 minutes from arrival to artery puncture, and no more than 110 minutes from arrival to reperfusion. The 2017 Standards and Guidelines Committee of the Society of NeuroInterventional Surgery ${ }^{78)}$ advocates ideal treatment times of no more than 30 minutes from arrival to the start of imaging, no more than 60 minutes from arrival to artery puncture, and no more than 90 minutes from arrival to reperfusion.

When MT is selected, treatment must be started as soon as possible, and recanalization must be achieved as soon as possible with the optimal approach [Grade A]. When MT cannot be promptly administered, the patient must be transported to the nearest medical institution capable of providing MT [Grade B]. Moreover, it is critical that sites offering MT establish a care system for shortening the times from arrival to imaging, from imaging to puncture, from puncture to recanalization, and from onset to reperfusion. When IV rt-PA has been administered, the start of MT must not be delayed for the evaluation of thrombolysis effectiveness [Grade D].

\subsection{Device Selection \\ (Recommendation)}

As first-line devices for MT, stent retrievers and aspiration catheters have been shown to have comparable efficacy [Grade A]. Although a combined technique with a stent retriever and an aspiration catheter may be considered, it must be realized that this technique has not been shown to produce superior outcomes, involves complicated techniques, and incurs high costs [Grade C1].

Among the RCTs that showed the efficacy of MT, SWIFT-PRIME, EXTEND-IA, and REVASCAT were limited to the Solitaire FR, and DAWN was limited to the Trevo Pro, whereas stent retrievers were used in many of the other trials, with the exception of THERAPY.

The Contact Aspiration vs. Stent Retriever for Successful Revascularization (ASTER) study ${ }^{79}$ was an RCT that compared first-line ADAPT with an aspiration catheter and first-line use of a stent retriever. A total of 381 patients with acute ischemic stroke within 6 hours of onset featuring occlusion of the intracranial ICA or the M1 or M2 segment of the MCA were randomized to an ADAPT group (192 patients) or a stent retriever group (189 patients). The primary endpoint of good recanalization (TICI grade $2 b-3$ ) at the end of treatment was achieved by $85.4 \%$ of the patients in the ADAPT group and $83.1 \%$ of the patients in the stent retriever group (OR: 1.20; 95\% CI: $0.68-2.10 ; p=0.53$ ). There was no significant difference. Similarly, no significant difference was seen in the change in the NIHSS score after 24 hours and in the outcome at 90 days, but superiority was not demonstrated. In the Comparison of Direct 
Aspiration vs. Stent Retriever as a First Approach (COMPASS) trial, which involved 270 patients with acute ischemic stroke due to occlusion from the distal ICA to the MCA bifurcation within 6 hours of onset, the recanalization rate of TICI $\geq 2 \mathrm{~b}$ did not differ in the ADAPT group, at $91.7 \%$, and the stent retriever group, at $89 \%(p=0.54)$, and the patients with a good outcome at 90 days (mRS score 0-2) were comparable, at $52 \%$ vs. $49 \%$, which demonstrated non-inferiority. ${ }^{80)}$ The above evidence shows that, as first-line devices for MT, stent retrievers and aspiration catheters have comparable efficacy. [Grade A]. Thrombectomy using a stent retriever, however, has a more rapid learning curve than ADAPT. ${ }^{81)}$

The Penumbra Separator 3D trial ${ }^{82)}$ was an RCT that compared concomitant treatment with the Penumbra 3D stent retriever (not approved in Japan) and a Penumbra catheter with ADAPT. The primary outcome measure was the rate of recanalization with an mTICI score of $\geq 2 \mathrm{a}$, which was achieved by $87.2 \%$ and $82.3 \%$ of the patients, respectively (difference 4.9 percentage points, $90 \%$ CI -3.6 to 13.5 percentage points), and an mRS score of $0-2$ at 90 days was achieved by $45.3 \%$ and $45.8 \%$ of the patients, which showed the treatment to be non-inferior. The ASTER2 trial (ClinicalTrials.gov Identifier: NCT03290885), which compared a combined technique with a stent retriever and aspiration catheter to stent retriever therapy alone, showed no difference in the TICI 2c-3 effective recanalization rate between the two. Although this combined technique has been shown to have a benefit ${ }^{83,84)}$ and may be considered for first-line treatment, it must be realized that this technique has not been shown to produce superior outcomes, involves complicated techniques, and incurs high costs [Grade C1].

\subsection{Anesthesia Selection \\ (Recommendation)}

MT is generally performed under local anesthesia. General anesthesia may be considered if medically required or if it can be provided without delaying the treatment [Grade C1].

A meta-analysis by HERMES of the effects of general anesthesia in seven studies ${ }^{85)}$ compared measures in 893 patients in a medical therapy group, 236 patients in a general anesthesia group, and 561 patients in a non-general anesthesia (sedation) group. Thrombectomy showed significantly better outcomes than medical therapy both with general anesthesia (adjusted common OR: 1.52, 95\% CI: 1.09-2.11) and without (adjusted common OR: 2.33, 95\% CI: 1.75-3.10), but the unanesthetized patients had significantly better outcomes than those who received general anesthesia (adjusted common OR: 1.53, 95\% CI: 1.14-2.04).
The Sedation vs. Intubation for Endovascular Stroke Treatment (SIESTA) ${ }^{86)}$ study, which was an RCT that compared general anesthesia with sedation, was conducted to confirm the hypothesis that sedation is superior to general anesthesia in early neurological improvement in patients undergoing thrombectomy, but no differences were seen in the change in the NIHSS score at 24 hours, which was -3.2 in the general anesthesia group and -3.6 in the sedation group, for a mean intergroup difference of -0.4 (95\% CI: -3.4 to 2.7 ). The proportion of patients achieving an mRS score of $0-2$ at 90 days was significantly higher in the general anesthesia group $(37.2 \%$ vs. $18.2 \% \mathrm{p}=0.01)$. The hypothesis of Anesthesia During Stroke (AnStroke), ${ }^{87)}$ a similar RCT, was "anesthesia technique does not have an impact on neurological outcome after this procedure, as long as severe hypotension during the procedure is avoided." With blood pressure strictly controlled, the rate of an mRS score of 0-2 at 90 days was $42.2 \%$ in the general anesthesia group and $40.0 \%$ in the sedation group, meaning that the outcomes did not differ. The hypothesis of the General or Local Anesthesia in Intra Arterial Therapy (GOLIATH) ${ }^{88}$ trial postulated that "patients who were under conscious sedation would have less infarct growth." The general anesthesia group, however, had a high recanalization rate of $\mathrm{mTICI} \geq 2 \mathrm{~b}(76.9 \%$ vs. $60.3 \%$, $\mathrm{p}=0.04$ ) and tended to have smaller infarct volume growth (median $8.2 \mathrm{~mL}$ vs. $19.4 \mathrm{~mL}$ in the sedation group, $\mathrm{p}=0.10$ ). Moreover, $\mathrm{mRS}$ scores at 90 days were significantly better (adjusted common OR: 1.91, 95\% CI: 1.03-3.56), and the proportion of patients with an mRS score of 0-2 at 90 days tended to be higher (OR: 1.90, 95\% CI: 0.93-3.90). A meta-analysis of the data of these three studies ${ }^{89}$ showed that, in 368 patients with large artery occlusion of the anterior circulation and NIHSS score $\geq 10$ (mean age 71.5 years, median NIHSS 17), a significantly higher recanalization rate of TICI $\geq 2 \mathrm{~b}$ (72.7\% vs. $63.2 \%$, aOR: $1.84,95 \%$ CI: $1.12-3.01)$ and better outcomes (adjusted common OR of better mRS score 1.58, 95\% CI: 1.09-2.29; mRS score of 0-2 at 90 days: $49.2 \%$ vs. $35.1 \%$, aOR: $2.16,95 \%$ CI: 1.31-3.54) were achieved in the general anesthesia group. Although the time from arrival to puncture was longer in the general anesthesia group (75 minutes vs. 69 minutes, $\mathrm{p}=0.04$ ), the times from puncture to recanalization (51.5 minutes vs. 70.5 minutes, $\mathrm{p}=0.15$ ) and arrival to recanalization (150 minutes vs. 165 minutes, $\mathrm{p}=0.86$ ) were not.

In the seven studies included in the HERMES meta-analysis, the large selection bias associated with frequent administration of general anesthesia as a medical indication may have resulted in the 
significant relationship between general anesthesia and poor MT outcomes. In SIESTA, AnStroke, and GOLIATH, in contrast, quickly inducing general anesthesia to minimize the time to puncture and careful intraoperative management including of blood pressure appears to have contributed to better outcomes associated with higher recanalization rates, but these three studies were single-center studies, and only one used clinical outcome as the primary endpoint.

In conclusion, although MT is generally administered under local anesthesia, general anesthesia may be considered if medically required or if it can be provided without delaying the treatment [Grade C1].

\subsection{Informed Consent}

(Recommendation)

The benefits and risks of MT should be explained and informed consent obtained from eligible patients or their legal representatives.

Because MT caries the risk of causing intracranial hemorrhage as a complication, for eligible patients, the patient or his or her legal representative should preferably be informed of and consent to the anticipated benefits and risks of MT before treatment is begun. There may be situations in clinical practice, however, in which the patient lacks decision-making ability and no legal representative is present. Justifications for medical acts include the presence of a medical indication, professional qualification, and the consent of the patient, but in emergency situations, a medical act may be performed without consent (i.e., is legal) if medically required and performed by a qualified professional (presumed consent). ${ }^{90-92)}$

Taking enough time to fully inform the patient who will undergo MT can be difficult because the time from onset to the start of the procedure greatly affects improvements in functional outcomes. Patients, moreover, are often unable to understand what is said or are unable to give consent, and a legal representative often cannot be found in time.

Since MT is medically required and will be performed by a qualified professional according to the above recommended treatment indications, MT for acute ischemic stroke caused by an ICA or MCA M1 segment occlusion can be justified without consent when the patient lacks decision-making ability and no legal representative is present. ${ }^{78)}$ Medical institutions should establish a policy about whether to allow MT based on their recent treatment outcomes for situations where no legal representative is present. With such a policy in place, treatment in the absence of a legal representative is allowable only when the practitioner decides, after a consensus-based decision involving members of the medical care team, that, for the particular patient, performing MT is clearly more beneficial than not performing MT. This approach allows rational decision-making without relying solely on the independent judgment of the treating physician. Even so, attempts should be made after the start of treatment to contact a legal representative as soon as possible to explain the rationale of the team for providing treatment. This recommendation is informed by a policy on the careful administration of intravenous alteplase advocated by the Research on Bioethics in Severe Stroke group ${ }^{93)}$ in Japan and is presented here with the consent of the members of the Guidelines authoring committee. When the above recommended treatment indications are not satisfied, proactive actions should be taken, such as referring the matter to the ethics committee of the medical institution in question.

\section{Environment for MT}

\subsection{Required elements for medical institutions (Recommendation) \\ The site should have an environment that allows IV rt-PA and should be equipped with an angio-suite that allows MT to be performed at any time [Grade $\mathrm{A}$ ].}

Given that MT is endovascular therapy in which a thrombectomy device is guided to an intracranial artery, sites must have an environment that allows neuroendovascular therapy, that is, be equipped with an angiography room or an angio-suite in operating rooms and have a stroke physician able to start acute treatment as soon as possible after arrival. Sites, moreover, should preferably monitor and disclose information about their environment, constantly working to shorten the time to reperfusion.

IV rt-PA should be considered first-line therapy for acute occlusion of major cerebral arteries. It should be performed at sites that are capable of providing head CT or MRI, general hematology tests, coagulation tests, and electrocardiography, that have an acute stroke care specialist able to begin treating patients as soon as possible after arrival, and that have a system in place to promptly provide neurosurgical intervention if needed.

\subsection{Operator Certification \\ (Recommendation) \\ MT must be performed by a specialist in neuro- endovascular therapy or by a certified MT operator with comparable experience.}

Given that MT involves guiding a thrombectomy device to an intracranial artery, operators must 
attend training sessions to learn how to use each device they intend to use so that they can safely provide MT. Training session attendees must be specialists in neuroendovascular therapy certified by the JSNET or a certified MT operator with comparable experience. Experience comparable to that of a specialist in neuroendovascular therapy is generally comparable to having passed the qualification exam for the specialist, and doctors who have such experience and satisfy the following criteria become certified MT operators upon registering with the JSNET.

- Is a member of the JSNET

- Is specialized in one of four core disciplines (i.e., neurosurgery, internal medicine, radiology, and emergency medicine)

- Has performed diagnostic cerebral angiography for at least 200 patients

- Has performed neuroendovascular therapy for 100 patients (including 20 as the first operator and 15 as a provider of MT)

- Participates in the continuing education program of and renews registration with the JSNET once every 5 years

\section{Status of MT}

Scientific evidence has been presented that adding MT to medical therapy that includes IV rt-PA improves the outcomes of patients with acute ischemic stroke caused by large artery occlusion within 6 hours of onset. It has also been shown that performing MT within 16 or 24 hours improves functional outcomes in patients with acute ischemic stroke caused by large artery occlusion substantially after the time last known well. These findings, however, involve patients diagnosed with large artery occlusion of the anterior circulation (ICA or M1 segment of the MCA) and are found eligible for treatment by diagnostic imaging, and the outcomes were achieved because MT primarily using stent retrievers was quickly performed. It must be realized that sufficient knowledge has yet to be accumulated to establish the efficacy of MT in certain patient populations, and that the approval conditions of all thrombectomy devices other than the Trevo and Solitaire in Japan call for use in acute ischemic stroke within 8 hours of onset. To establish the efficacy and safety of endovascular therapy using intracranial thrombectomy devices in acute ischemic stroke, practicing physicians have a duty to continuously and actively assist in postmarketing surveillance and clinical research.

MT offers efficacy superior to medical therapy when performed under a care system that allows MT to be started and recanalization to be achieved as soon as possible, with proper patient selection, and by a properly trained practitioner according to the specified procedures. All those involved in acute ischemic stroke care must fulfill their roles and build systems for providing care to ensure good patient outcomes.

\section{Acknowledgments}

When revising these Guidelines, the authors greatly benefited from the specialist opinions of Dr. Akira Ishii (Department of Neurosurgery, Kyoto University), Dr. Hirotoshi Imamura and Dr. Nobuyuki Sakai (Department of Neurosurgery, Kobe City Medical Center General Hospital), Dr. Yuji Matsumaru (Division of Stroke Prevention and Treatment, Department of Neurosurgery, University of Tsukuba), and Dr. Shinichi Yoshimura (Department of Neurosurgery, Hyogo College of Medicine). 


\section{Conflicts of Interest Disclosure}

\begin{tabular}{|l|c|c|c|c|c|c|c|c|c|c|c|c|}
\hline FY 2017-2019 & 1 & 2 & 3 & 4 & 5 & 6 & 7 & 8 & 9 & 10 & 11 & 12 \\
\hline Hiroshi Yamagami & $/$ & $/$ & $/$ & $\mathrm{B}$ & $/$ & $\mathrm{A}$ & $/$ & $/$ & $/$ & $/$ & $/$ & $/$ \\
\hline Mikito Hayakawa & $/$ & $/$ & $/$ & $/$ & $/$ & $/$ & $/$ & $/$ & $/$ & $/$ & $/$ & $/$ \\
\hline Manabu Inoue & $/$ & $/$ & $/$ & $/$ & $/$ & $/$ & $/$ & $/$ & $/$ & $/$ & $/$ & $/$ \\
\hline Koji Iihara & $/$ & $/$ & $/$ & $\mathrm{A}$ & $/$ & $/$ & $\mathrm{A}$ & $/$ & $/$ & $/$ & $/$ & $/$ \\
\hline Kuniaki Ogasawa & $/$ & $/$ & $/$ & $/$ & $/$ & $/$ & $/$ & $/$ & $/$ & $/$ & $/$ & $/$ \\
\hline Kazunori Toyoda & $/$ & $/$ & $/$ & $\mathrm{A}$ & $/$ & $/$ & $/$ & $/$ & $/$ & $/$ & $/$ & $/$ \\
\hline Yasuhiro Hasegawa & $/$ & $/$ & $/$ & $\mathrm{A}$ & $/$ & $/$ & $/$ & $/$ & $/$ & $/$ & $/$ & $/$ \\
\hline Kenji Ohata & $/$ & $/$ & $/$ & $/$ & $/$ & $/$ & $/$ & $/$ & $/$ & $/$ & $/$ & $/$ \\
\hline Yoshiaki Shiokawa & $/$ & $/$ & $/$ & $/$ & $/$ & $\mathrm{A}$ & $\mathrm{A}$ & $/$ & $/$ & $/$ & $/$ & $/$ \\
\hline Kazuhiko Nozaki & $/$ & $/$ & $/$ & $\mathrm{A}$ & $/$ & $/$ & $\mathrm{A}$ & $/$ & $/$ & $/$ & $/$ & $/$ \\
\hline Masayuki Ezura & $/$ & $/$ & $/$ & $/$ & $/$ & $/$ & $/$ & $/$ & $/$ & $/$ & $/$ & $/$ \\
\hline Toru Iwama & $/$ & $/$ & $/$ & $\mathrm{A}$ & $/$ & $\mathrm{A}$ & $\mathrm{A}$ & $/$ & $/$ & $/$ & $/$ & $/$ \\
\hline
\end{tabular}

\section{Items for COI self-disclosure}

1. Reporting criterion for being a director or consultant of a corporation or for-profit group:

Annual compensation from single corporation/group

A: $\geq 1$ million yen, $\mathrm{B}: \geq 5$ million yen, $\mathrm{C}: \geq 10$ million yen

2. Ownership of stock

Reporting criterion: Annual stock-based profit (sum of dividends and profit from sale) for a single corporation

A: $\geq 1$ million yen, B: $\geq 5$ million yen, C: $\geq 10$ million yen, D: own $\geq 5 \%$ of all shares

3. Patent royalty from a corporation or for-profit group

Reporting criteria: Annual patent royalty per patent

A: $\geq 1$ million yen, $B: \geq 5$ million yen, $\mathrm{C}: \geq 10$ million yen

4. Daily allowance (e.g., speaker fees) received for the time/work of the investigator for meeting attendance (e.g., presenting, advising) from a corporation or for-profit group

Reporting criteria: Annual total of daily allowance (e.g., speaker fees) from single corporation/group

A: $\geq 500,000$ yen, B: $\geq 1$ million yen, C: $\geq 2$ million yen

5. Manuscript fees received for authoring manuscripts or pamphlets from a corporation or for-profit group

Reporting criteria: Annual total of manuscript fees from single corporation/group

A: $\geq 500,000$ yen, B: $\geq 1$ million yen, C: $\geq 20$ million yen

6. Research fees provided under a contract by a corporation or for-profit group

Reporting criterion: Total amount allocated, from a single corporation/group, to the performance of contractual research for which the reporter has substantial decision-making authority in terms of medical research ((1) joint research, (2) contract research, (3) clinical trial, (4) other)

A: $\geq 1$ million yen, $\mathrm{B}: \geq 5$ million yen, $\mathrm{C}: \geq 10$ million yen

7. Scholarship (grant) provided by a corporation or for-profit group

Reporting criterion: Total amount actually allocated by a single corporation/group in the form of a scholarship (grant) to a purpose for which the reporter has substantial decision-making authority to the reporter or the department/field or laboratory of the reporter

A: $\geq 1$ million yen, $\mathrm{B}: \geq 5$ million yen, $\mathrm{C}: \geq 10$ million yen

8. If the reporter is the head of a university department sponsored through a grant from a corporation or for-profit group

Reporting criterion: Does the annual per-corporation amount actually allocated as a donation to a purpose for which the reporter has substantial decision-making authority exceed 1 million yen? A: Yes, B: No 
9. Other compensation (e.g., travel/gifts not directly related to research)

Reporting criterion: Annual compensation from single corporation/group

A: $\geq 50,000$ yen, B: $\geq 200,000$ yen, C: $\geq 500,000$ yen

Reporter's spouse, relative in the first degree or closer, or person sharing income/wealth

10. Director or consultant of a corporation or for-profit group

Reporting criterion: Annual compensation from single corporation/group

A: $\geq 1$ million yen, B: $\geq 5$ million yen, C: $\geq 10$ million yen

11. Ownership of stock

Reporting criterion: Annual stock-based profit (sum of dividends and profit from sale) for a single corporation

A: $\geq 1$ million yen, B: $\geq 5$ million yen, C: $\geq 10$ million yen, D: own $\geq 5 \%$ of all shares

12. Patent royalty from a corporation or for-profit group

Reporting criteria: Annual patent royalty per patent

A: $\geq 1$ million yen, $\mathrm{B}: \geq 5$ million yen, $\mathrm{C}: \geq 10$ million yen 


\section{References}

1) Endo K, Koga M, Sakai N, et al.: Stroke outcomes of Japanese patients with major cerebral artery occlusion in the post-alteplase, pre-MERCI era. $J$ Stroke Cerebrovasc Dis 22: 805-810, 2013

2) National Institute of Neurological Disorders and Stroke rt-PA Stroke Study Group: Tissue plasminogen activator for acute ischemic stroke. $N$ Engl J Med 333: 1581-1587, 1995

3) Emberson J, Lees KR, Lyden P, et al.: Effect of treatment delay, age, and stroke severity on the effects of intravenous thrombolysis with alteplase for acute ischaemic stroke: a meta-analysis of individual patient data from randomised trials. Lancet 384: 1929-1935, 2014

4) Jauch EC, Saver JL, Adams HP, et al.: Guidelines for the early management of patients with acute ischemic stroke: a guideline for healthcare professionals from the American Heart Association/American Stroke Association. Stroke 44: 870-947, 2013

5) Shinohara Y, Ogawa A, Suzuki N, et al.: The 2009 Guidelines for the Management of Stroke. 2009 (Japanese)

6) Hacke W, Kaste M, Bluhmki E, et al.: Thrombolysis with alteplase 3 to 4.5 hours after acute ischemic stroke. N Engl J Med 359: 1317-1329, 2008

7) Guideline Committee of the Japan Stroke Society for the intravenous rt-PA (alteplase) in acute ischemic stroke. Guidelines for intravenous application of rt-PA (alteplase), October 2012, partly revised in September 2016. Jpn J Stroke 39: 43-86, 2017 (Japanese)

8) Furlan A, Higashida R, Wechsler L, et al.: Intra-arterial prourokinase for acute ischemic stroke. The PROACT II study: a randomized controlled trial. Prolyse in Acute Cerebral Thromboembolism. JAMA 282: 2003-2011, 1999

9) Ogawa A, Mori E, Minematsu K, et al.: Randomized trial of intraarterial infusion of urokinase within 6 hours of middle cerebral artery stroke: the middle cerebral artery embolism local fibrinolytic intervention trial (MELT) Japan. Stroke 38: 2633-2639, 2007

10) Smith WS, Sung G, Starkman S, et al.: Safety and efficacy of mechanical embolectomy in acute ischemic stroke: results of the MERCI trial. Stroke 36: 1432-1438, 2005

11) Smith WS, Sung G, Saver J, et al.: Mechanical thrombectomy for acute ischemic stroke: final results of the Multi MERCI trial. Stroke 39: 1205-1212, 2008

12) Bose A, Henkes H, Alfke K, et al.: The Penumbra System: a mechanical device for the treatment of acute stroke due to thromboembolism. AJNR Am J Neuroradiol 29: 1409-1413, 2008

13) Penumbra Pivotal Stroke Trial Investigators: The penumbra pivotal stroke trial: safety and effectiveness of a new generation of mechanical devices for clot removal in intracranial large vessel occlusive disease. Stroke 40: 2761-2768, 2009

14) Saver JL, Jahan R, Levy EI, et al.: Solitaire flow restoration device versus the Merci Retriever in patients with acute ischaemic stroke (SWIFT): a randomised, parallel-group, non-inferiority trial. Lancet 380: 1241-1249, 2012

15) Nogueira RG, Lutsep HL, Gupta R, et al.: Trevo versus Merci retrievers for thrombectomy revascularisation of large vessel occlusions in acute ischaemic stroke (TREVO 2): a randomised trial. Lancet 380: 12311240, 2012

16) Broderick JP, Palesch YY, Demchuk AM, et al.: Endovascular therapy after intravenous t-PA versus t-PA alone for stroke. N Engl J Med 368: 893-903, 2013

17) Ciccone A, Valvassori L, Nichelatti M, et al.: Endovascular treatment for acute ischemic stroke. $N$ Engl J Med 368: 904-913, 2013

18) Kidwell CS, Jahan R, Gornbein J, et al.: A trial of imaging selection and endovascular treatment for ischemic stroke. N Engl J Med 368: 914-923, 2013

19) Chimowitz MI: Endovascular treatment for acute ischemic stroke--still unproven. $N$ Engl J Med 368: 952-955, 2013

20) Berkhemer OA, Fransen PS, Beumer D, et al.: A randomized trial of intraarterial treatment for acute ischemic stroke. N Engl J Med 372: 11-20, 2015

21) Goyal M, Demchuk AM, Menon BK, et al.: Randomized assessment of rapid endovascular treatment of ischemic stroke. N Engl J Med 372: 1019-1030, 2015

22) Campbell BC, Mitchell PJ, Kleinig TJ, et al.: Endovascular therapy for ischemic stroke with perfusionimaging selection. N Engl J Med 372: 1009-1018, 2015

23) Saver JL, Goyal M, Bonafe A, et al.: Stent-retriever thrombectomy after intravenous t-PA vs. t-PA alone in stroke. N Engl J Med 372: 2285-2295, 2015

24) Nogueira RG, Jadhav AP, Haussen DC, et al.: Thrombectomy 6 to 24 hours after stroke with a mismatch between deficit and infarct. N Engl J Med 378: 11-21, 2018

25) Albers GW, Marks MP, Kemp S, et al.: Thrombectomy for stroke at 6 to 16 hours with selection by perfusion imaging. N Engl J Med 378: 708-718, 2018

26) Guideline Committee of the Japan Stroke Society for the management of stroke. The 2015 Guidelines for the Management of Stroke [Supplement 2019]. 2019 (Japanese)

27) Guideline Committee of the Japan Stroke Society for the intravenous rt-PA (alteplase) in acute ischemic stroke. Guidelines for Intravenous Thrombolysis (Recombinant Tissue-type Plasminogen Activator), the Third Edition, March 2019:. Jpn J Stroke 41: 205-246, 2019 (Japanese)

28) Toyoda K, Koga M, Iguchi Y, et al.: Guidelines for intravenous thrombolysis (recombinant tissue-type plasminogen activator), the Third Edition, A Guideline from the Japan Stroke Society. Neurol Med Chir (Tokyo) March 2019:; 59: 449-491, 2019.

29) Turk AS, Spiotta A, Frei D, et al.: Initial clinical experience with the ADAPT technique: a direct aspiration 
first pass technique for stroke thrombectomy. $J \mathrm{Neu}$ rointerv Surg 6: 231-237, 2014

30) Turk AS, Frei D, Fiorella D, et al.: ADAPT FAST study: a direct aspiration first pass technique for acute stroke thrombectomy. J Neurointerv Surg 6: 260-264, 2014

31) Castaño C, Serena J, Dávalos A: Use of the new solitaire (TM) AB device for mechanical thrombectomy when merci clot retriever has failed to remove the clot. A Case Report. Interv Neuroradiol 15: 209-214, 2009

32) Zaidat OO, Castonguay AC, Gupta R, et al.: North American Solitaire Stent Retriever Acute Stroke registry: post-marketing revascularization and clinical outcome results. J Neurointerv Surg 6: 584-588, 2014

33) Mueller-Kronast NH, Zaidat OO, Froehler MT, et al.: Systematic evaluation of patients treated with neurothrombectomy devices for acute ischemic stroke: primary results of the STRATIS registry. Stroke 48: 2760-2768, 2017

34) Zaidat OO, Castonguay AC, Nogueira RG, et al.: TREVO stent-retriever mechanical thrombectomy for acute ischemic stroke secondary to large vessel occlusion registry. J Neurointerv Surg 10: 516-524, 2018

35) Binning MJ, Bartolini B, Baxter B, et al.: Trevo 2000: Results of a large real-world registry for stent retriever for acute ischemic stroke. J Am Heart Assoc 7: e010867, 2018

36) Sakai N, Ota S, Matsumoto Y, et al.: Efficacy and safety of REVIVE SE thrombectomy device for acute ischemic stroke: River JAPAN (Reperfuse Ischemic Vessels with Endovascular Recanalization Device in Japan). Neurol Med Chir (Tokyo) 58: 164-172, 2018

37) Zaidat OO, Bozorgchami H, Ribó M, et al.: Primary results of the multicenter ARISE II study (analysis of revascularization in ischemic stroke with EmboTrap). Stroke 49: 1107-1115, 2018

38) Kaneko N, Komuro Y, Yokota H, Tateshima S: Stent retrievers with segmented design improve the efficacy of thrombectomy in tortuous vessels. J Neurointerv Surg 11: 119-122, 2019

39) Sakai N, Imamura H, Adachi H, et al.: First-in-man experience of the Versi Retriever in acute ischemic stroke. J Neurointerv Surg 11: 296-299, 2019

40) van den Berg LA, Dijkgraaf MG, Berkhemer OA, et al.: Two-year outcome after endovascular treatment for acute ischemic stroke. N Engl J Med 376: 1341-1349, 2017

41) Jovin TG, Chamorro A, Cobo E, et al.: Thrombectomy within 8 hours after symptom onset in ischemic stroke. N Engl J Med 372: 2296-2306, 2015

42) Goyal M, Menon BK, van Zwam WH, et al.: Endovascular thrombectomy after large-vessel ischaemic stroke: a meta-analysis of individual patient data from five randomised trials. Lancet 387: 1723-1731, 2016

43) Bracard S, Ducrocq X, Mas JL, et al.: Mechanical thrombectomy after intravenous alteplase versus alteplase alone after stroke (THRACE): a randomised controlled trial. Lancet Neurol 15: 1138-1147, 2016
44) Mocco J, Zaidat OO, von Kummer R, et al.: Aspiration thrombectomy after intravenous alteplase versus intravenous alteplase alone. Stroke 47: 2331-2338, 2016

45) Muir KW, Ford GA, Messow CM, et al.: Endovascular therapy for acute ischaemic stroke: the Pragmatic Ischaemic Stroke Thrombectomy Evaluation (PISTE) randomised, controlled trial. J Neurol Neurosurg Psychiatry 88: 38-44, 2017

46) Albers GW, Marks MP, Kemp S, et al.: Thrombectomy for stroke at 6 to 16 hours with selection by perfusion imaging. N Engl J Med 378: 708-718, 2018

47) Hacke W, Kaste M, Fieschi C, et al.: Intravenous thrombolysis with recombinant tissue plasminogen activator for acute hemispheric stroke. The European Cooperative Acute Stroke Study (ECASS). JAMA 274: 1017-1025, 1995

48) Wardlaw JM, Mielke O: Early signs of brain infarction at CT: observer reliability and outcome after thrombolytic treatment--systematic review. Radiology 235: 444-453, 2005

49) Albers GW, Thijs VN, Wechsler L, et al.: Magnetic resonance imaging profiles predict clinical response to early reperfusion: the diffusion and perfusion imaging evaluation for understanding stroke evolution (DEFUSE) study. Ann Neurol 60: 508-517, 2006

50) Inoue $M$, Mlynash M, Straka M, et al.: Patients with the malignant profile within 3 hours of symptom onset have very poor outcomes after intravenous tissue-type plasminogen activator therapy. Stroke 43: 2494-2496, 2012

51) Sanák D, Nosál' V, Horák D, et al.: Impact of diffusion-weighted MRI-measured initial cerebral infarction volume on clinical outcome in acute stroke patients with middle cerebral artery occlusion treated by thrombolysis. Neuroradiology 48: 632-639, 2006

52) Haussen DC, Dehkharghani S, Rangaraju S, et al.: Automated CT perfusion ischemic core volume and noncontrast CT ASPECTS (Alberta Stroke Program Early CT Score): correlation and clinical outcome prediction in large vessel stroke. Stroke 47: 2318-2322, 2016

53) Demeestere J, Garcia-Esperon C, Garcia-Bermejo P, et al.: Evaluation of hyperacute infarct volume using ASPECTS and brain CT perfusion core volume. Neurology 88: 2248-2253, 2017

54) de Margerie-Mellon C, Turc G, Tisserand M, et al.: Can DWI-ASPECTS substitute for lesion volume in acute stroke? Stroke 44: 3565-3567, 2013

55) Terasawa Y, Kimura K, Iguchi Y, et al.: Could clinical diffusion-mismatch determined using DWI ASPECTS predict neurological improvement after thrombolysis before 3 h after acute stroke? J Neurol Neurosurg Psychiatry 81: 864-868, 2010

56) Yoshimoto $T$, Inoue $M$, Yamagami H, et al.: Use of diffusion-weighted imaging-alberta stroke program early computed tomography score (DWI-ASPECTS) and ischemic core volume to determine the malignant profile in acute stroke. J Am Heart Assoc 8: e012558, 2019

57) Bouslama M, Bowen MT, Haussen DC, et al.: Selection paradigms for large vessel occlusion acute ischemic 
stroke endovascular therapy. Cerebrovasc Dis 44: 277-284, 2017

58) Rebello LC, Bouslama M, Haussen DC, et al.: Stroke etiology and collaterals: atheroembolic strokes have greater collateral recruitment than cardioembolic strokes. Eur J Neurol 24: 762-767, 2017

59) Román LS, Menon BK, Blasco J, et al.: Imaging features and safety and efficacy of endovascular stroke treatment: a meta-analysis of individual patient-level data. Lancet Neurol 17: 895-904, 2018

60) Campbell BCV, Majoie CBLM, Albers GW, et al.: Penumbral imaging and functional outcome in patients with anterior circulation ischaemic stroke treated with endovascular thrombectomy versus medical therapy: a meta-analysis of individual patient-level data. Lancet Neurol 18: 46-55, 2019

61) Kakita H, Yoshimura S, Uchida K, et al.: Impact of endovascular therapy in patients with large ischemic core: subanalysis of recovery by endovascular salvage for cerebral ultra-acute embolism Japan Registry 2. Stroke 50: 901-908, 2019

62) Haussen DC, Lima FO, Bouslama M, et al.: Thrombectomy versus medical management for large vessel occlusion strokes with minimal symptoms: an analysis from STOPStroke and GESTOR cohorts. J Neurointerv Surg 10: 325-329, 2018

63) Goyal N, Tsivgoulis G, Malhotra K, et al.: Medical management vs mechanical thrombectomy for mild strokes: An International Multicenter Study and systematic review and meta-analysis. JAMA Neurol 77: 16-24, 2019

64) Sarraj A, Sangha N, Hussain MS, et al.: Endovascular therapy for acute ischemic stroke with occlusion of the middle cerebral artery M2 segment. JAMA Neurol 73: 1291-1296, 2016

65) Menon BK, Hill MD, Davalos A, et al.: Efficacy of endovascular thrombectomy in patients with M2 segment middle cerebral artery occlusions: metaanalysis of data from the HERMES Collaboration. $J$ Neurointerv Surg 11: 1065-1069, 2019

66) Miura M, Yoshimura S, Sakai N, et al.: Endovascular therapy for middle cerebral artery M2 segment occlusion: subanalyses of RESCUE-Japan Registry 2. J Neurointerv Surg 11: 964-969, 2019

67) Phan K, Phan S, Huo YR, Jia F, Mortimer A: Outcomes of endovascular treatment of basilar artery occlusion in the stent retriever era: a systematic review and meta-analysis. J Neurointerv Surg 8: 1107-1115, 2016

68) Writing Group for the BASILAR Group, Zi W, Qiu Z, et al: Assessment of endovascular treatment for acute basilar artery occlusion via a Nationwide Prospective Registry. JAMA Neurol 77: 561-573, 2020

69) Liu X, Dai Q, Ye R, et al.: Endovascular treatment versus standard medical treatment for vertebrobasilar artery occlusion (BEST): an open-label, randomised controlled trial. Lancet Neurol 19: 115-122, 2020

70) Emberson J, Lees KR, Lyden P, et al.: Effect of treatment delay, age, and stroke severity on the effects of intravenous thrombolysis with alteplase for acute ischaemic stroke: a meta-analysis of individual patient data from randomised trials. Lancet 384: 1929-1935, 2014

71) Yamaguchi T, Mori E, Minematsu K, et al.: Alteplase at $0.6 \mathrm{mg} / \mathrm{kg}$ for acute ischemic stroke within 3 hours of onset: Japan Alteplase Clinical Trial (J-ACT). Stroke 37: 1810-1815, 2006

72) Mistry EA, Mistry AM, Nakawah MO, et al.: Mechanical thrombectomy outcomes with and without intravenous thrombolysis in stroke patients: a meta-analysis. Stroke 48: 2450-2456, 2017

73) Suzuki K, Kimura K, Takeuchi M, et al.: The randomized study of endovascular therapy with versus without intravenous tissue plasminogen activator in acute stroke with ICA and M1 occlusion (SKIP study). Int J Stroke 14: 752-755, 2019

74) Campbell BCV, Mitchell PJ, Churilov L, et al.: Tenecteplase versus alteplase before thrombectomy for ischemic stroke. N Engl J Med 378: 1573-1582, 2018

75) Campbell BCV, Mitchell PJ, Churilov L, et al.: Effect of intravenous tenecteplase dose on cerebral reperfusion before thrombectomy in patients with large vessel occlusion ischemic stroke: The EXTEND-IA TNK Part 2 Randomized Clinical Trial. JAMA 323: 1257-1265, 2020

76) Thomalla G, Simonsen CZ, Boutitie F, et al.: MRI-guided thrombolysis for stroke with unknown time of onset. N Engl J Med 379: 611-622, 2018

77) Saver JL, Goyal M, van der Lugt A, et al.: Time to treatment with endovascular thrombectomy and outcomes from ischemic stroke: a meta-analysis. JAMA 316: 1279-1288, 2016

78) McTaggart RA, Ansari SA, Goyal M, et al.: Initial hospital management of patients with emergent large vessel occlusion (ELVO): report of the standards and guidelines committee of the Society of NeuroInterventional Surgery. J Neurointerv Surg 9: 316-323, 2017

79) Lapergue B, Blanc R, Gory B, et al.: Effect of endovascular contact aspiration vs stent retriever on revascularization in patients with acute ischemic stroke and large vessel occlusion: The ASTER Randomized Clinical Trial. JAMA 318: 443-452, 2017

80) Turk AS, Siddiqui A, Fifi JT, et al.: Aspiration thrombectomy versus stent retriever thrombectomy as firstline approach for large vessel occlusion (COMPASS): a multicentre, randomised, open label, blinded outcome, non-inferiority trial. Lancet 393: 998-1008, 2019

81) Nishi H, Ishii A, Nakahara I, et al.: Different learning curves between stent retrieval and a direct aspiration first-pass technique for acute ischemic stroke. J Neurosurg 129: 1456-1463, 2018

82) Nogueira RG, Frei D, Kirmani JF, et al.: Safety and efficacy of a 3-dimensional stent retriever with aspiration-based thrombectomy vs aspiration-based thrombectomy alone in acute ischemic stroke intervention: a randomized clinical trial. JAMA Neurol 75: 304-311, 2018

83) McTaggart RA, Tung EL, Yaghi S, et al.: Continuous aspiration prior to intracranial vascular embolectomy (CAPTIVE): a technique which improves outcomes. J Neurointerv Surg 9: 1154-1159, 2017 
84) Tomasello A, Ribò M, Gramegna LL, et al.: Procedural approaches and angiographic signs predicting firstpass recanalization in patients treated with mechanical thrombectomy for acute ischaemic stroke. Interv Neuroradiol 25: 491-496, 2019

85) Campbell BCV, van Zwam WH, Goyal M, et al.: Effect of general anaesthesia on functional outcome in patients with anterior circulation ischaemic stroke having endovascular thrombectomy versus standard care: a meta-analysis of individual patient data. Lancet Neurol 17: 47-53, 2018

86) Schönenberger S, Uhlmann L, Hacke W, et al.: Effect of conscious sedation vs general anesthesia on early neurological improvement among patients with ischemic stroke undergoing endovascular thrombectomy: a randomized clinical trial. JAMA 316: 1986-1996, 2016

87) Löwhagen Hendén P, Rentzos A, Karlsson JE, et al.: General anesthesia versus conscious sedation for endovascular treatment of acute ischemic stroke: The AnStroke Trial (Anesthesia During Stroke). Stroke 48: 1601-1607, 2017

88) Simonsen CZ, Yoo AJ, Sørensen LH, et al.: Effect of general anesthesia and conscious sedation during endovascular therapy on infarct growth and clinical outcomes in acute ischemic stroke: a randomized clinical trial. JAMA Neurol 75: 470-477, 2018
89) Schönenberger S, Hendén PL, Simonsen CZ, et al.: Association of general anesthesia vs procedural sedation with functional outcome among patients with acute ischemic stroke undergoing thrombectomy: a systematic review and meta-analysis. JAMA 322: 1283-1293, 2019

90) Masunari M: Medical treatment when the consent of the patient/family cannot be confirmed. Japan Medical Journal 4756: 66, 2016 (Japanese)

91) Chian W: The adult guardian's right in medical interventions. Takaoka Law Review 29: 39-74, 2011 (Japanese)

92) WMA Declaration of Lisbon on the rights of the patient; 2005, https://www.wma.net/policies-post/wma-declaration-of-lisbon-on-the-rights-of-the-patient/.

93) Miyamoto S, Ida R, Minematsu K, et al.: A report from the Research on Bioethics in Severe Stroke research group; 2012 (Japanese).

Corresponding author: Hiroshi Yamagami, MD, PhD Department of Stroke Neurology, National Hospital Organization Osaka National Hospital, 2-1-14 Hoenzaka, Chuo-ku, Osaka 540-0006, Japan. e-mail: yamagami-brain@umin.ac.jp; yamagami.hiroshi@ncvc.go.jp 